\title{
An Automated Evaluation Procedure for Artificial Imagination Systems
}

\author{
by \\ Can Serif Mekik
}

A thesis submitted to the Faculty of Graduate and Post Doctoral Affairs in partial fulfillment of the requirements for the degree of

\section{Master of Cognitive Science}

\author{
Carleton University \\ Ottawa, ON \\ (C) 2016 \\ Can Serif Mekik
}




\begin{abstract}
Artificial imagination systems are systems which perform tasks that, if performed by humans, would require imagination. Evaluation is a pervasive methodological challenge for artificial imagination systems research. Consequently it is difficult to compare imagination systems and to generalize results.

Methodological challenges associated with imagination system evaluation can be addressed through the implementation of artificial assessors. The goal of the present work is to demonstrate that well-motivated artificial assessors for imagination systems can be developed and to make a case that an evaluation methodology incorporating such artificial assessors will be an improvement on current practices in the field. I establish a theoretical framework for the development of such automatic assessors based on the philosophy and psychology of imagination, and I report on an experiment investigating the performance of a pilot automatic assessor in the domain of texture synthesis.
\end{abstract}




\section{Acknowledgements}

I would like to thank my supervisor, Jim Davies, my committee members, Kasia Muldner and Anthony Whitehead, and my internal external reviewer, David Mould, for their many contributions to the final product that is this document. If it were not for their input, encouragement and support the document you are about to read would be far inferior to what it is now. I would also like to thank Ida Toivonen and Raj Singh. I have learned much through my collaborations with them, and much of that knowledge has informed this work.

Thanks to Luke Kersten, Mike Vertolli, Gautam Sukumar, Drew Blackmore, Ehsan Amjadian and, especially, Naba Ahsan for their friendship, emotional support and stimulating discussion. I am going to miss all the discussion, commiseration and shenanigans. I am very grateful to Paul and Ayşe Heinbecker, as well as Selin and Yasemin Heinbecker, who have become like family to me over the course of my studies. Finally, I would like to thank my parents, Çiğdem and Cem Mekik, my brother, Deniz Mekik, my aunts, Figen Mekik and Meltem Yllmaz, as well as Serap Mekik, Emel Tunçbilek, Ümit Ağan, Lemis Benderlioğlu, Yıldız Gürbüz and the rest of my family for supporting me throughout the course of this work. Without their encouragement, advice and support, this thesis would have been but a dream. 


\section{Table of Contents}

1 Introduction 1

1.1 Imagination in Artificial Intelligence . . . . . . . . . . . . . . 1

1.1.1 Applications for Imagination Systems Research . . . . . . . . 2

1.1 .2 Modeling Imagination . . . . . . . . . . . . . . . . . . 3

1.2 Evaluation: A Challenge for Imagination Systems Research . . . . . . 4

1.2.1 Patterns and Problems ............... 5

1.2.2 Research Question ................. 7

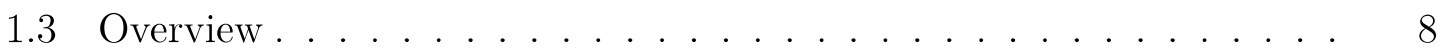

2 Imagination Systems 10

2.1 Imagination in Philosophy and Psychology . . . . . . . . . . . . . . 10

2.1.1 Representational Features of Imaginings . . . . . . . . . . . . 11

2.1.2 Imagination Viewed as a Process . . . . . . . . . . . . . . . . 12

2.2 Defining Imagination Tasks . . . . . . . . . . . . . . . . . . . . 13

2.2.1 Imaginative Prompts . . . . . . . . . . . . . . . . . 14

2.2 .2 Imaginative Elaboration _ . . . . . . . . . . . . . . . . . 15

2.2.3 Comparison to Other Definitions ........... 16

2.3 Closing Remarks . . . . . . . . . . . . . . . . . . . 18 
3 A Theory of Imagination System Correctness 19

3.1 An Analysis of Imagination Task Demands . . . . . . . . . . . . . . . 20

3.1 .1 How Not to Evaluate Imaginings . . . . . . . . . . . . . . . . 20

3.1.2 Prompts and Elaborative Choices: Keys to Evaluation . . . . 21

3.2 Developing Scoring Functions . . . . . . . . . . . . . . . . 22

3.2.1 Setting the Stage . . . . . . . . . . . . . 22

3.2.2 Correctness as Compliance with Principles of Generation . . . 23

3.3 Closing Remarks . . . . . . . . . . . . . . . 25

4 Mirroring Compliance Assessment (MCA) 26

4.1 The Mirroring Principle . . . . . . . . . . . . . . . 26

4.1.1 What is the Mirroring Principle? . . . . . . . . . . . . . 27

4.1.2 Some Psychological Evidence . . . . . . . . . . . . . . 29

4.1.3 Why Mirroring Is Relevant . . . . . . . . . . . . . . . 31

4.2 MCA Automation . . . . . . . . . . . . . . . . 32

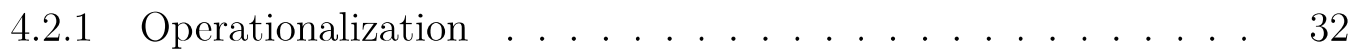

4.2.2 Mirroring and Binary Classification . . . . . . . . . . . 33

4.2.3 A Restriction On Training Data . . . . . . . . . . . . 33

4.2.4 MCA as a One-Class Classification Task . . . . . . . . . . 35

4.3 Closing Remarks . . . . . . . . . . . . . . . . 37

5 Pilot MCA: Design and Methods 38

5.1 Overview of Experiment . . . . . . . . . . . . . . 38

5.1.1 Experimental Setting . . . . . . . . . . . . 39

5.1 .2 Methodology ...................... 40 
5.2 Experimental Stimuli . . . . . . . . . . . . . . . . . . . . 41

5.2 .1 TIPS-2b Images . . . . . . . . . . . . . . . . . . . . . 43

$5.2 .2 \quad$ Image Preprocessing . . . . . . . . . . . . . . . . . . 44

5.2 .3 Stimulus Preparation . . . . . . . . . . . . . . . . . 44

5.3 Human Assessor Data Collection . . . . . . . . . . . . . . 46

5.3 .1 Participants . . . . . . . . . . . . . . 46

5.3.2 Stimuli and Materials . . . . . . . . . . . . . . 47

5.3 .3 Design . . . . . . . . . . . . . . . . . . . . 47

5.3 .4 Procedure . . . . . . . . . . . . . . . . . . . 47

5.4 Assessor Implementation . . . . . . . . . . . . . . . . . . . 48

$5.4 .1 \quad$ Design . . . . . . . . . . . . . . . . . . . . . . . 49

5.4 .2 Training Procedure . . . . . . . . . . . . . . . . 51

5.4 .3 Running the Test Images . . . . . . . . . . . . . . . . . 53

5.5 Closing Remarks . . . . . . . . . . . . . . . . . . 53

6 Pilot MCA: Results and Discussion $\quad 54$

6.1 Overview of Generalizability Theory and Agreement Statistics . . . 54

$6.1 .1 \quad$ Computing $\mathbf{E} \rho^{2}$ and $\Phi \ldots \ldots \ldots \ldots \ldots$

6.2 Results . . . . . . . . . . . . . . . . . . . . 58

6.2 .1 Analysis of Human Judgments . . . . . . . . . . . . . . 59

6.2.2 Comparison of Human-Machine and Human-Human Pairwise Agreement Scores . . . . . . . . . . . . . . . . 61

6.3 Discussion . . . . . . . . . . . . . . . . . . . . 65

6.3 .1 Human Judgment Data . . . . . . . . . . . . . . . 65

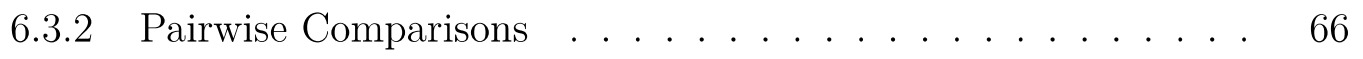


6.3.3 Conclusions ........................ 68

6.4 Closing Remarks . . . . . . . . . . . . . . . . . 69

$\begin{array}{lll}7 & \text { Conclusion } & 70\end{array}$

7.1 General Discussion ... . . . . . . . . . . . . . . 71

7.2 Future Work . . . . . . . . . . . . . . . . . . . . . 74

$\begin{array}{ll}\text { Bibliography } & 76\end{array}$

$\begin{array}{ll}\text { Appendices } & 83\end{array}$ 


\section{List of Tables}

5.1 Texture Categories Represented in the TIPS-2b Dataset . . . . . . 43 


\section{List of Figures}

5.1 Sample Images from the Test Set. . . . . . . . . . . . . . . . . . . 45

5.2 Reproduction of an Experimental Trial. . . . . . . . . . . . . . . . . 48

5.3 High-level View of Automatic Assessor Behavior. . . . . . . . . . . . 49

5.4 RNN Architecture . . . . . . . . . . . . . . . . 50

6.1 Image Acceptance Rates for Human assessors by Image Source . . . . 59

6.2 Human Image Acceptance Rates by Prompt . . . . . . . . . . . . . . 60

6.3 Human-Machine and Human-Human Pairwise Agreement Scores . . . 62

6.4 Human-Machine and Human-Human Relative Pairwise Agreement by

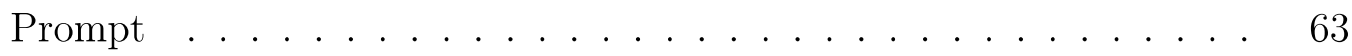

6.5 Human-Machine and Human-Human Absolute Pairwise Agreement by

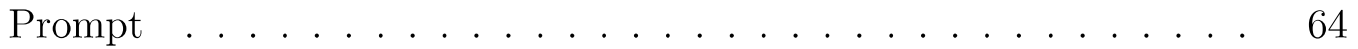




\section{List of Appendices}

A Briefing Text and Comprehension Questions 


\section{Chapter 1 Introduction}

Imagination systems are systems which perform tasks that, if performed by humans, would require imagination. For instance, imagine a restaurant. What color are its interior walls? How big is its dining area? What kind of food does it serve? If you have answers to these questions you have created an imagining. In other words, you have performed a task which requires imagination: the task of imagining a restaurant. It is possible for an artificial intelligence to perform similar tasks. Such systems are artificial imagination systems. For example, the Science of Imagination Laboratory Imagination Engine (SOILIE; Breault, Ouellet, Somers, \& Davies, 2013; Vertolli et al., 2014) generates novel visual scenes containing some target everyday object when prompted with a noun denoting that object.

\subsection{Imagination in Artificial Intelligence}

Discussion or reports of imagination systems date back, at least, to 1962 (see Morton, 1962/2000). However, imagination systems research is not conducted by a unified community. On the one hand, there is a small group of researchers who are interested in modeling or implementing imagination. On the other hand, there are subfields of computer science and artificial intelligence that are dedicated to specific varieties of imagination task for various applications. Before I introduce the main topic of this 
thesis, which is the development of automated evaluation procedures for artificial imagination systems, I would like to give the reader a sense of the stakes involved in imagination systems research.

\subsubsection{Applications for Imagination Systems Research}

Perhaps the most obvious application for artificial imagination systems is in art and design. Procedural content generation and evolutionary art are two subfields of artificial intelligence which explore imagination systems' potential for such applications. According to Togelius, Yannakakis, Stanley, and Browne (2011), "procedural content generation (PCG) refers to creating game content automatically, through algorithmic means". Game designers may use PCG for game elements such as "terrain, maps, levels, stories, dialogue, quests, characters, rulesets, dynamics and weapons" (Togelius et al., 2011). Similarly, evolutionary art combines "evolutionary techniques and computer graphics to create artistic images of great complexity" (Lewis, 2008). Techniques associated with PCG and evolutionary art have also been experimentally applied to industrial design tasks with some success (e.g. Hornby, 2003; Vico, Veredas, Bravo, \& Almaraz, 1999).

Though art and design are intuitively salient applications for imagination systems, these systems are applicable in a much larger set of domains. Let me illustrate with some examples. Roy, Hsiao, and Mavridis (2004) implemented a manipulator robot which interprets natural language commands by imagining a scene from the user's visual perspective. Thomee, Huiskes, Bakker, and Lew (2007, 2008) implemented

an imagination system that improves interactive image search by synthesizing recommendations that are representative of database content relevant to user queries. Chella, Frixione, and Gaglio (2005) present a robot which can use its imagination to 
plan routes as it navigates its environment. Finally, Hallin et al. (2009) experimented with the use of imagination in the control of autonomous underwater vehicles in mine countermeasure missions.

\subsubsection{Modeling Imagination}

As the review above suggests, imagination systems are applicable in domains as diverse as art, design, machine meta-cognition, interactive search, navigation and problem solving. The diversity of applications for imagination systems can partially be explained by the role of imagination in cognition. Current theories about the cognitive role of imagination suggest that imagination supports abilities such as meta-cognition, fictional and moral reasoning, and counterfactual reasoning (for a review, see Gendler, 2013). Imagination supports other cognitive abilities by giving agents access to useful information that is not readily available in the environment or in memory. Of course, this generalization grossly simplifies the complex relationship between imagination and other cognitive abilities, but it is useful to keep in mind when considering the appeal of imagination systems in applied settings.

Imagination systems research can be a useful tool for developing and refining theories about human imagination and its interface with other cognitive abilities. That is to say, though imagination systems are often modeled on human imagination, they can also serve as models of human imagination. To illustrate this point, recall SOILIE (Breault et al., 2013; Vertolli et al., 2014). Unlike previous work in modeling mental imagery, which focuses on the role of mental images in problem solving (e.g. Kosslyn \& Schwartz, 1977; Tabachneck-Schijf, Leonardo, \& Simon, 1997), work on SOILIE is dedicated to the development of a theory of the processes which enable the generation of realistic mental images. Work on a sub-system of SOILIE suggests 
that realistic visual mental images of objects belonging to an unfamiliar category can be formed by transferring visuospatial knowledge from objects belonging to similar, but familiar, categories (Gagne \& Davies, 2013; Somers, Gagné, Astudillo, \& Davies, 2011). This finding corroborates empirical work by Ward (1994) which showed that category structure plays an important role in the generation of imaginings. As more sophisticated imagination systems are developed, they may shed a brighter light on the processes that govern human imagination.

\subsection{Evaluation: A Challenge for Imagination Systems Research}

Let us move on to the topic of the present work. A methodological problem pervades imagination systems research: imagination system evaluation. Many imagination systems researchers lament that exisiting imagination system evaluation procedures are unsatisfactory or underdeveloped (Galanter, 2010; Setchi, Lagos, \& Froud, 2007; Togelius et al., 2011). Challenges in imagination system evaluation lead to difficulties in validating and comparing imagination systems. Furthermore, imagination systems often follow design patterns which call for the evaluation of imaginings as part of their normal operation.

A common design pattern in evolutionary art (see Lewis, 2008) can serve as a good example of the prominence of evaluation in some imagination system architectures. Evolutionary art systems first generate an initial set of artifacts and refine them through a process of recombination and elimination. Artifacts are tested against some fitness function; high performers are allowed to have descendants while low performers are discarded. The choice of fitness function for such a design pattern has significant impact on the overall quality of the system. Fitness functions can be formally defined, but the best results are often achieved by having human raters 
judge creations for fitness (Lewis, 2008).

\subsubsection{Patterns and Problems}

It is important to remark that imagination system evaluation is recognized to be a difficult task (Lewis, 2008; Togelius et al., 2011). It is often noted that one of the main challenges in implementing imagination systems is the choice of evaluation metrics (e.g., Galanter, 2010; Lewis, 2008; Togelius et al., 2011; Vico et al., 1999). Imagination tasks are paradigmatic examples of ill-defined problems. Ill-defined problems are characterized by unclear success conditions, multiple acceptable solutions and im-

plicit or unclear constraints (Lynch, Ashley, Pinkwart, \& Aleven, 2009). As such it is difficult to identify stable imagination task demands in order to develop evaluation procedures.

A consequence of the difficulty associated with evaluating imagination systems is that imagination systems are often simply not evaluated in any formal capacity. When evaluation is attempted, on the other hand, procedures tend to be ad hoc in that they tend to be based on the specific task or application to which an imagination system is being applied rather than on general considerations about what an imagination system is supposed to do. Let me present a few examples of such evaluation procedures.

Visuo is the component of SOILIE which is responsible for generating visuospatial data to be used in the formation of a mental image (Somers et al., 2011; Gagne \& Davies, 2013). As discussed above, Visuo is able to transfer knowledge from familiar objects to unfamiliar objects. Somers et al. (2011) evaluated Visuo's knowledge transfer procedure by varying the similarity of the source of knowledge transfer from the target and assessing the accuracy of predictions made as a result of knowledge transfer. 
Aleksander, Dunmall, and Del Frate (1999) investigated imagination systems tasked with visualizing novel combinations of object features based on past experience. For example, having seen a yellow triangle and a red circle an imaginer would be tasked with visualizing a yellow circle. Upon being presented with the test prompt, imaginers would instantiate responses in a visual memory store. For evaluation, noise in the visual store during the imagination task is compared to noise during perception.

Stening, Jacobsson, and Ziemke (2005) investigated a simple robot's ability to anticipate changes to its sensor array as it moves through its environment on a fixed path. The robot has a very small set of concepts (3 or 5 depending on the trial) and attempts to anticipate which concept will be active some distance into the future. Stening et al. (2005) compare the sequence of active concepts over the length of the experiment to the sequence of concepts observed during navigation.

In the publications discussed above, though evaluation procedures are justified in the immediate experimental context, little to no effort is made to tie the procedures in to more general concerns about imagination. For instance, Somers et al. (2011) simply state that it is desirable for Visuo's knowledge transfer procedure to result in accurate imaginings. This statement makes sense in the context of the chosen task, which is to use imagination to make up for a lack of knowledge, but it isn't clear what exactly a success for Visuo in this context reveals about it as a model of imagination in general. Put in general terms, ad hoc evaluation procedures make it difficult to interpret and generalize results and to compare imagination systems.

In applications such as art or media, imagination systems can be evaluated based on ratings provided by human assessors. For instance, Norton, Heath, and Ventura (2013) ask human assessors to rate the creations of DARCI, an artificial artist, for 
creativity. In cases where humans are qualified to serve as assessors, this approach can yield good results. This is why humans are often preferred to automatic methods for artifact evaluation in evolutionary art (Lewis, 2008). When human assessors are available, they may allow researchers to bypass some of the problems associated with interpreting results from ad hoc evaluation procedures.

Despite offering some advantages, the use of human assessors for imagination system evaluation has significant drawbacks. First, human assessors are not applicable to all imagination tasks. For instance, it would be difficult to find qualified human assessors for the imaginings produced by Stening et al.'s (2005) imaginer robots. Second, recruiting and running human participantts in an imagination system evaluation procedure is resource intensive.

\subsubsection{Research Question}

To summarize, on the one hand, ad hoc evaluation procedures lead to difficulties in interpreting and generalizing results, as well as comparing imagination systems, and, on the other hand, sometimes it is possible to bypass these challenges by running human participants, but it this approach is not generally applicable and it is resource intensive. Well-motivated evaluation procedures for artificial imagination systems could address the challenges presented by existing approaches to imagination system evaluation. But, how can we systematically develop well-motivated evaluation procedures for imagination systems? The answer I defend in the present text is that we should commit to developing automatic imagination system assessors. 


\subsection{Overview}

The goal of the present work is to demonstrate that well-motivated artificial assessors for imagination systems can be developed, and to make a case that an evaluation methodology incorporating such artificial assessors will be an improvement on current practices in the field.

The philosophy and psychology of imagination can guide us in the enterprise of developing automatic imagination system evaluation procedures. I discuss how the philosophy and psychology of imagination can inform this enterprise in Chapters 2 and 3. In particular, I present a methodological framework for imagination system evaluation procedure development based on these fields. At the core of this proposal are two concepts: a precise definition for imagination tasks (Chapter 2) and Walton's (1990) concept of a principle of generation (Chapter 3).

In Chapters 2 and 3, I argue that imagination tasks are tasks in which imaginers generate imaginings by elaborating on imaginative prompts. Imaginings are revealing of the elaborative choices made by imaginers, and it is these elaborative choices that are subject to evaluation. In particular, elaborative choices are constrained by locally applicable social conventions - that is, by principles of genearation. The upshot of this observation is that artificial imagination system assessors can be developed by identifying and operationalizing compliance with relevant principles of generation.

Armed with the theoretical framework outlined above, the rest of my work is dedicated to the development and implementation of a generic evaluation procedure for imagination systems. This evaluation procedure operationalizes compliance with a principle of generation that is applicable by default, called the principle of mirroing. This principle and its operationalization are introduced in Chapter 4. Chapter 5 and 
6 report on a pilot experiment to develop and validate an artificial assessor in the domain of texture synthesis. Chapter 7 concludes with a general discussion of the proposed approach to imagination system evaluation and of the pilot project. 


\section{Chapter 2 Imagination Systems}

In the previous chapter, I defined imagination systems as systems which perform imagination tasks - tasks that, if performed by humans, would require imagination. In order to develop a methodology for the systematic evaluation and comparison of imagination systems, we must clearly state the task demands associated with imagination tasks. A precise and explicit definition of imagination tasks will prove to be an invaluable tool in this enterprise.

There are some attempts to define imagination tasks in the literature. These definitions are often modeled on mental imagery tasks, though some authors prefer to think of imagination tasks as a species of design task. In this chapter, I present a new definition of imagination tasks which is grounded in the philosophy and psychology of imagination and which improves on existing definitions. In Section 2.1, I review relevant literature from the philosophy and psychology of imagination, and I highlight two significant generalizations. In Section 2.2 I formulate and discuss my definition.

\subsection{Imagination in Philosophy and Psychology}

The term imagination has many usages and, for this reason, many authors believe that it is difficult to define (Gendler, 2013). For instance, Stevenson (2003) enumerates a dozen usages of the term and makes no claim to exhaustivity. In cognitive 
science, however, the term's usage is fairly consistent; it picks out a class of phenomena no more ill-defined than the likes of perception, memory, or problem solving. According to this usage, the term imagination refers to an agent's ability to produce a distinctive set of representations, sometimes called imaginings (Currie \& Ravenscroft, 2002; Gendler, 2013; Davies, Atance, \& Martin-Ordas, 2011). ${ }^{1}$

\subsubsection{Representational Features of Imaginings}

Imaginings share some features with other mental representations such as beliefs, desires, percepts, plans, and memories. These similarities are sometimes expressed by suggesting that imaginings are belief-like, percept-like, and so on (Currie \& Ravenscroft, 2002). Ordinarily, mental representations are thought to have at least two components: an attitude and a content (Fodor, 1985/1994). The content of a mental representation corresponds to the state of affairs picked out by that representation, whereas its attitude corresponds to its cognitive role. The attitude-content analysis is applicable to imaginings.

Let us discuss imaginative mental attitudes in some more detail. Mental attitudes are distinguished based on the functional roles they play in cognition, including their representational roles. In this respect, imaginings differ from other mental representations in an important capacity. Beliefs, desires, percepts, plans, and memories are all representational states which can roughly be classified into two groups based on the way they relate to actual states of affairs. Beliefs, percepts, and memories represent actual states of affairs as they are or as they were. Desires and plans, on the other hand, represent states of affairs as they should be. These two sets of representations

\footnotetext{
${ }^{1}$ The term imagination is sometimes used synonymously with creativity, however imagination and creativity are distinct concepts. This text is focused exclusively on imagination. For a discussion of the relationship between imagination and creativity, see Gaut (2003).
} 
are bound by different norms. For example, false beliefs ought be abandoned whereas unsatisfied desires ought be pursued. These differences are expressed by stating that the two groups have different directions of fit-different norms govern how they relate to actual states of affairs (Humberstone, 1992). It is often noted that, unlike other mental representations, imaginings are not bound by any direction of fit (Urmson, 1967; Walton, 1990; Humberstone, 1992; Currie \& Ravenscroft, 2002).

\subsubsection{Imagination Viewed as a Process}

So far, we have discussed representational features of imaginings, but there is also significant agreement about some features of the processes that generate imaginings. Nichols and Stich (2000) propose a cognitive theory of pretense that is informed by the experimental literature. Their theory explicitly incorporates many elements common to other theories in the literature and, for this reason, it forms the core of the present discussion.

According to Nichols and Stich (2000), an episode of pretense begins with an initial premise, hereafter called a prompt. The prompt is placed in a special memory mechanism that Nichols and Stich call the possible world box (PWB). As an episode of pretense develops, further representations are added to the PWB. These representations together form "an increasingly detailed representation of what the world would be like if the initiating representation were true" (Nichols \& Stich, 2000). Needless to say, the contents of the PWB are imaginings.

The process of filling in unspecified details of the episode of pretense is termed elaboration. Nichols and Stich (2000) suggest that imaginative elaboration is driven by agents' knowledge of the world and of the information relevant to an episode of pretense. The agent uses representations in the PWB to inform its behavior dur- 
ing the episode of pretense, and as the episode unfolds, the PWB is updated: new representations are added and obsolete representations are deleted.

Mental imagery research offers a similar perspective. Humans can form mental images on the basis of both verbal and non-verbal, depictive prompts (e.g., Kosslyn, Thompson, Sukel, \& Alpert, 2005). Note the additional detail that imaginative prompts may be descriptive (language-like) or depictive (picture-like). Consider observing a sketch of an apple, if in looking at that sketch you imagine it to be red or green or any other colour you have created an imagining based on a depictive prompt. Let us call the theory that the process of generating imaginings is one of elaborating on prompts, the prompt elaboration theory.

\subsection{Defining Imagination Tasks}

The review above suggests the following: the philosophy and psychology of imagination is host to two significant generalizations about imagination. Notably, (i) imaginings have no direction of fit and (ii) imaginings are generated by a process which elaborates on an initial prompt. Let us define imagination tasks on the basis of these generalizations as follows.

(D) An imagination task is a task in which an agent forms an imagining, which is a representation with no direction of fit, through a process of elaboration. Imagination tasks are initiated by imaginative prompts, and imaginings are elaborations on their respective imaginative prompts.

Below I discuss the notions of imaginative prompt and imaginative elaboration in further detail. In the next chapter, these notions will prove important. 


\subsubsection{Imaginative Prompts}

To see how imaginative prompts operate, let us consider some examples. Both supposition and visual mental imagery tasks can be defined by suitably chosen prompts. For instance, in ordinary language, a supposition is initiated by an utterance of the form shown in (1a). We can gloss (1a) as an order to imagine something in a certain way, as in (1b).
(1) a. Suppose $X$.
b. Imagine $X$ in a belief-like manner.

Likewise, visual mental images can be initiated by an utterance of the form (2a) which can be glossed as shown in $(2 \mathrm{~b})$.

(2) a. Visualize $X$.

b. Imagine $X$ in a vision-like manner.

Notice that in both cases, I render imaginative prompts with the imperative form (3), which glosses a more ordinary way of asking someone to imagine something.

\section{(3) Imagine $p$.}

I am not committed to the specifics of the individual glosses. One may, for example, argue that there is more to supposing than just imagining in a belief-like manner. This is not a debate I wish to engage, as it is of no consequence to the present discussion. Instead, I would like to draw attention to the fact that in rendering (1a) and (2a) using form (3), we can see that each utterance specifies two important parameters for the respective episodes of imagination. The first of these parameters is the content of the episode, represented by the placeholder $X$, and the second is the manner in which the imagining is to be instantiated - whether it is language-like or perception 
like, for example. The specification of these two parameters is the defining feature of imaginative prompts.

\subsubsection{Imaginative Elaboration}

Let us now discuss the notion of imaginative elaboration. Nichols and Stich (2000) define elaboration as a process which fills in details left unspecified by an imaginative prompt. However, an alternative conceptualization will prove useful. To develop this conceptualization, we need only note that, given the way Nichols and Stich define elaboration, imaginings have contents that are more specific than, but consistent with, the contents of their prompts.

Consider the following prompt.

(4) Visualize a wild tiger.

Upon complying with (4), you may have answers to some of the questions in (5), but not to others.

(5) a. What parts of the tiger are visible to you?

b. How big is the tiger?

c. What color is the tiger's fur?

d. What is the tiger's posture?

e. How many stripes does the tiger have?

f. Is the tiger close to any other animals?

In so far as you have answers to at least some of these questions, you have elaborated on prompt (4): you have created an imagining whose content is not only a tiger, but a tiger that has properties that agree with your responses to the questions in (5). 
Each of your responses can be contrasted with alternative possible responses. You happen to have given the responses you did in contrast to the alternatives because, in forming your imagining, you made some elaborative choices-contributions to an episode of imagination not specified by the imaginative prompt that contrast with a set of salient alternatives. ${ }^{2}$

In summary, imaginative elaborations are the elaborative choices imaginers make in producing imaginings. In other words, an imagining can be thought of as a prompt along with a set of elaborative choices. For artificial imagination systems, elaborative choices are choices systems make with respect to some relevant feature space. For example, an artificial imagination system that performs (4), may output a labeled raster image. Since a variety of raster images are deserving of the label "wild tiger", the choice of a particular raster on the part of the imagination system is an elaborative choice.

\subsubsection{Comparison to Other Definitions}

Mental imagery systems are often described as having the ability to synthesize images based on an input specifying what the image should be about (e.g., Breault et al., 2013; Heath, Dennis, \& Ventura, 2015; Thomee et al., 2008). Sometimes, formal characterizations of imagery tasks are provided (see Murray, 2007; Vertolli \& Davies, 2014b). When imagery tasks are defined formally, it is done by analogy to classification tasks: given a label $\ell$, imagination systems are required to provide a point in some feature space which should be labeled $\ell$. Though it is primarily designed to characterize mental imagery tasks, this approach can be extended to imagination

\footnotetext{
${ }^{2}$ Lest the terminology be misleading, I do not take elaborative choices to necessarily be deliberate choices.
} 
tasks that are unrelated to mental imagery (Heath et al., 2015).

An alternative approach, embraced in Davies et al. (2011), is to define imagination tasks by analogy to design tasks. This approach calls attention to features of imagination tasks that are obscured in the formal approaches discussed above. In particular, it calls attention to the fact that imaginings are constructed to fit a certain purpose or satisfy a certain goal, and to the fact that imaginers have the power to choose the features that their imaginings have.

Definition (D) can be seen as a refinement on existing approaches to imagination task definition. The notion of elaboration on a prompt is consistent with both the design-based approach to imagination task definition and the classification-based approach to imagination task definition. Moreover, definition (D) distinguishes imagination tasks from other tasks. Definition (D) is a refinement on existing approaches to imagination task definition in that it is a fairly precise statement grounded in the psychology and philosophy of imagination that can explain intuitions about imaginings and about imagination tasks.

For instance, search task can be described as task in which a system is required to return points in some feature space which deserve some given label $\ell$; however, search tasks are not imagination tasks. This is due to the fact that search tasks admit a direction of fit: in a search task one looks for a correct solution to some search query. This is not the case in an imagination task; however this is not to say that search engines cannot be implemented using imagination systems, as the work of (Thomee et al., 2008) shows.

The refinement with respect to design tasks is of a similar flavor. A design is something that is created in the service of a goal-a set of specifications. Consequently 
it is possible for the design to fail to meet its goal. It is worth noting that while it makes sense to talk of unacceptable imaginings, it is awkward to speak of failed or mistaken imaginings. Definition (D) can help us explain the intuitions behind these judgments. It does not make sense to talk of failed or mistaken imaginings because imaginings are not the kind of thing that can fail or be mistaken - they do not have directions of fit.

\subsection{Closing Remarks}

In this chapter, I defined imagination tasks as tasks in which agents must elaborate on imaginative prompts. This definition is promising as a general characterization of imagination tasks in that it follows directly from some generally accepted features of imagination, and in that it refines previous attempts to define imagination tasks. In the following chapter, this definition will prove to be a useful tool in dissecting imagination task demands in service of developing a methodological framework for systematic imagination system evaluation. 


\section{Chapter 3}

\section{A Theory of Imagination System Correctness}

Consider the imagination task prompted by (6).

(6) Imagine an apple.

While each imagining in (7), except for (7d), qualifies as an elaboration on (6), they give rise to contrasts.

(7) a. A red apple.

b. A bruised yellow apple.

c. ? A watermelon-sized, banana-shaped blue apple.

d. $\quad *$ A green banana.

Imaginings (7a) and (7b) appear to satisfy the task at hand, but (7c) is odd. In fact, it can be argued that imagining (7c) constitutes a failure to perform the task prompted by (6), even though there is nothing in the prompt that explicitly forbids such an imagining. Finally, (7d) unambiguously fails to satisfy the task prompted by (6).

In order to systematically evaluate and compare imagination systems, we need to capture contrasts of the sort above, and importantly, we need to do so in a principled manner. As discussed in Chapter 1, it is here that we are faced with a problem. The 
features that qualify imagination tasks as ill-defined problems are features that make it difficult to determine precise and general evaluation criteria.

In this chapter, I present the beginnings of a systematic theory of imagination system correctness. In Section 3.1, I argue that imagination systems should be evaluated on the quality of their elaborative choices. In Section 3.2, I discuss how general and systematic scoring procedures can be developed by appealing to Walton's (1990) notion of a principle of generation.

\subsection{An Analysis of Imagination Task Demands}

We can assess imaginings on a scale ranging from adequate to inadequate, as illustrated in the opening discussion of this chapter. What drives our assessments? And, what do our assessments reveal about imaginers? If we define imagination tasks in accordance with Definition (D), we can answer these questions.

Answers to the questions in the previous paragraph are commitments about imagination task demands - about what an imaginer must do in order to successfully perform an imagination task. An imagination system is correct to the extent that it performs imagination tasks well; so, commitments about imagination task demands translate naturally into a theory of imagination system correctness.

\subsubsection{How Not to Evaluate Imaginings}

Let us first discuss a tempting, but unworkable, approach to imagination system cor-

rectness. The content of a representation can be veridical (truthful or accurate) or erroneous (false or inaccurate), and a representation is (representationally) correct when its content is veridical. When the output of a piece of software is a representation, the software can often be assessed for correctness according to the represen- 
tational correctness of its output. For instance, a function designed to compute the $n$th Fibonacci number is only correct if, for every input representing an integer $n$, it returns a symbol that represents the $n$th Fibonacci number.

Imaginings are representations. It is, therefore, tempting to evaluate imaginings according to their representational correctness. However, imaginings cannot be assessed according to their representational correctness. By definition, if a representation can be evaluated on the grounds of its representational correctness, it has a direction of fit. But, as stated in Definition (D), imaginings have no direction of fit, which implies that they cannot be evaluated according to their representational correctness. Thus, what would be a convenient approach to imagination system evaluation is unavailable.

The restriction above seems to conflict with actual practice in imagination systems research. In particular, the restriction seems to be in direct conflict with the practices of research aiming to develop systems that generate realistic imaginings (e.g., Breault et al., 2013). I address this apparent paradox in the next chapter.

\subsubsection{Prompts and Elaborative Choices: Keys to Evaluation}

Definition (D) does not only help us identify unacceptable approaches to imagination system correctness, it also grounds a positive proposal. Recall that, according to Definition (D), an imagination task requires an imaginer to elaborate on an imaginative prompt. Consequently, an imaginer's contribution to an episode of imagination is defined by the elaborative choices made during that episode. An imagining is just the end result of the process of elaboration. Therefore, an assessment of the adequacy of an imagining is an indicator of the felicity of the elaborative choices that led to the construction of that imagining. 
Imagination tasks do not give imaginers unlimited freedom when it comes to elaborative choices. There are task specific constraints that imaginative elaborations must meet in order to be considered adequate. Some of these constraints are set by the imaginative prompt while others are set tacitly, as illustrated by $(7 \mathrm{~d})$ and $(7 \mathrm{c})$ respectively. An operational test that assesses the degree to which such constraints are met is an operational test of imagination system correctness.

\subsection{Developing Scoring Functions}

A consequence of the discussion above is that we can define systematic evaluation procedures for imagination systems in so far as we can systematically express the constraints imagination tasks place on imaginative elaborations. However, these constraints are elusive. While we can intuitively assess imaginings, an explicit theory is not available. In this section, I present a framework based on Walton's (1990) theory of representational art that is designed to facilitate the development of explicit theories of the constraints imagination tasks place on elaborative choices.

\subsubsection{Setting the Stage}

The category of representational art has fuzzy boundaries, but it includes artifacts such as paintings, novels, stories, plays, films and so on. Walton (1990) presents a theory of representational art and other related phenomena such as dreaming and day-dreaming. He suggests that much ground can be covered towards understanding representational art by pursuing an analogy to games of make-believe. Walton's notion of a game of make-believe is derivative of ordinary children's games, and is closely related to the notion of pretense discussed in Nichols and Stich (2000).

In any given game of make-believe, certain propositions are to be imagined. 
Walton (1990) calls these propositions fictional propositions. Fictional propositions establish a normative standard similar to, but distinct from, directions of fit. Participants in games of make-believe who do not imagine fictional propositions when the game calls for it are not playing the game appropriately (p. 39).

Objects in the real world can play one of three roles in games of make-believe: they can prompt imaginings, they can be the objects of imaginings or they can generate fictional propositions (Walton, 1990). Objects that generate fictional propositions are props. One of the most important claims of Walton's theory is that works of representational art can be analyzed as props in games of make-believe. Here, we will not explore the consequences of this claim, instead we will be more concerned with the mechanics of the generation of fictional propositions.

Props generate fictional propositions in accordance with principles of generation (Walton, 1990). Principles of generation are socially established rules that govern the generation of fictional propositions (Walton, 1990). Principles of generation may be established by means of explicit stipulation or they may be established implicitly, with no overt or deliberate formulation. Different principles may be applicable in different settings, depending on the participants of games of make-believe and on the games themselves.

\subsubsection{Correctness as Compliance with Principles of Generation}

There is a natural correspondence between elements of Walton's (1990) theory and those of Definition (D). Imaginative prompts are analogous to works of representational art, acceptable imaginings to the set of fictional propositions, and constraints imagination tasks place on imaginative elaborations to principles of generation. These correspondences are grounds for extending Walton's (1990) analysis of dreams and 
day-dreams (p. 43-51) to imaginings. An extension of the theory to imaginings leads an important hypothesis, let us call it $(\mathrm{H})$.

(H) Human assessments of imaginings are driven by principles of generation.

Hypothesis $(\mathrm{H})$ can ground a methodological proposal. We can think of imagination system correctness in terms of compliance with applicable principles of generation. We can then follow a three-step approach to imagination system evaluation. These steps are (i) to identify principles of generation applicable in imagination tasks of interest, (ii) to develop (or adapt) operational procedures that measure compliance with applicable principles, and (iii) to implement these procedures in order to obtain desired measurements of imagination system correctness.

The appeal of the proposed approach is that principles of generation are social constructs. That is to say, principles of generation are objects of study in their own right, and notably, they are theoretically independent of the circumstantial goals of individual imagination system development projects. Therefore, the identification and operationalization of principles of generation consists in a research program that can lead to the development of systematic and general evaluation procedures for imagination systems, and perhaps, may even improve our understanding of human imagination.

Identification and operationalization of applicable principles of generation promises to be a challenging enterprise (see Walton, 1990, Chapter 4). Presently, I do not have much to say in general about how imagination systems researchers should proceed in order to address these issues; my goal here is only to point out the possibility and to give a taste of its promise. However, a good place to start is to look at the philosophy and psychology of imagination. As we shall see, this literature already contains some 
promising candidates.

\subsection{Closing Remarks}

This chapter explored some consequences of defining imagination tasks in accordance with Definition (D). Definition (D) motivates a particular approach to imagination system evaluation. Imagination systems are to be evaluated on the appropriateness of their imaginative elaborations with respect to relevant imagination tasks. Imagination tasks place both implicit and explicit constraints on imaginative elaborations. Consequently, in order to develop systematic evaluation procedures for imagination systems, we must be able to systematically assess compliance with constraints imagination tasks place on imaginative elaborations.

I suggested, based on a review of Walton's (1990) theory of representational art, that the constraints imagination tasks place on imaginative elaborations are best thought of as principles of generation - social constructs that govern allowable imaginative elaborations. This suggestion led to a methodological proposal: systematic evaluation procedures for imagination systems can be developed through a research program that identifies and operationalizes applicable principles of generation. Here, operationalization is understood as the automation of applicable scoring procedures. In the next two chapters, I explore how evaluation procedures can be developed within the framework established here. 


\section{Chapter 4 Mirroring Compliance Assessment (MCA)}

Previous chapters were dedicated to a theoretical treatment of imagination tasks and their task demands. This investigation led to a methodological proposal for the development of systematic imagination system evaluation procedures. While it is possible to further refine the theoretical framework, we are now at a point where we can begin to develop evaluation procedures in accordance with the framework. In order to do so, we must identify a principle of generation, operationalize it, and ultimately implement an operational (and automatic) procedure for compliance measurement.

The present chapter addresses the first two of these steps. In particular, this chapter, is dedicated to the operationalization of a principle of generation, called the mirroring principle (Gendler, 2003).

\subsection{The Mirroring Principle}

Recall prompt (6) and imagining (7c).

(6) Imagine an apple.

(7c) ? A watermelon-sized, banana-shaped blue apple.

What is wrong with imagining (7c)? There are no watermelon-sized, banana-shaped blue apples - at least that is one way to express the intuition, but it risks obscuring the real issue with $(7 \mathrm{c})$. The problem with $(7 \mathrm{c})$ is not whether or not there are 
watermelon-sized, banana-shaped blue apples. If that were the issue, one should be able to rectify the oddness of $(7 \mathrm{c})$ by reassuring us that there are, in fact, watermelonsized, banana-shaped blue apples. However, I would not find such reassurance satisfying. Perhaps the existence of watermelon-sized, banana-shaped blue apples would render (7c) less offensive, but I would say that imaginings (7a) and (7b) still seem far superior.

(7a) A red apple.

(7b) A bruised yellow apple.

The mirroring principle is a principle of generation proposed by Gendler (2003) that can explain such judgments.

\subsubsection{What is the Mirroring Principle?}

Gendler (2003) describes the mirroring principle as follows:

Mirroring is manifest to the extent that features of [an] imaginary situation that have not been explicitly stipulated are derivable via features of their real-world analogues, or more generally, to the extent that imaginative content is taken to be governed by the same sorts of restrictions that govern believed content. (Gendler, 2003)

It is clear that the mirroring principle is not merely a descriptive principle, but rather a principle of generation, as flouting it amounts to generating a defective imagining:

The mirroring principle says that imagining and believing are attitudes with similar ranges of possible non-defective contents: the sorts of things that we are able to non-defectively imagine are the sorts of things that we could, in principle, non-defectively believe. (Gendler, 2003)

We can explain what is wrong with (7c) by appealing to the mirroring principle. Note that $(7 \mathrm{c})$ does not comply with the mirroring principle. Watermelon-sized, 
banana-shaped blue apples are highly unlikely occurrences in everyday experience (I have never seen or heard of such an apple). Given this, it is bizarre to infer from the premise that some object is an apple that it is watermelon-sized, banana-shaped, blue, or that it has any combination of these features. The elaborative choices that get us from (6) to (7c), however, correspond to such inferences, and thereby violate the mirroring principle.

There are some subtleties worth mentioning, however. Imaginings are not expected to mirror their real-world analogues perfectly. In fact, the extent to which imaginings have to be faithful to their real-world analogues appears to depend on the specifics of each episode of imagination. This phenomenon is called disparity (Gendler, 2003). Another subtlety is that the mirroring principle applies only to features that are not explicitly stipulated. Consider the prompt (8).

(8) Visualize a watermelon-sized, banana-shaped blue apple.

An imaginer would not violate the mirroring principle if they visualized some watermelonsized, banana-shaped, blue apple in response to (8), because this prompt explicitly calls for such an imagining. That is to say, the mirroring principle can be defeated if an imaginative prompt calls for an imagining that deviates from the kind of things that can be non-defectively believed. This is not to say that such prompts cancel the mirroring principle. More likely, prompts suspend the mirroring principle locally for the unusual contents that they call for.

For what follows, it will be helpful to summarize the mirroring principle with a short proposition. Gendler (2003) offers the following: "things that are [imagined] are the sorts of things that could be, in principle, believed." We can refine this proposition by making use of the language developed in previous chapters. I propose we adopt 
principle (M) as our working definition.

(M) Elaborative choices follow from plausible inferences on imaginative prompts.

The significance of the mirroring principle is threefold. First, there is some psychological evidence that humans comply with a principle of generation very much like the mirroring principle. Second, the mirroring principle explains how imagination can support simulation-based cognitive abilities, as many authors think imagination does. Finally, as discussed above, the principle is almost universally applicable as it is applicable by default.

\subsubsection{Some Psychological Evidence}

Gendler (2003) formulates the mirroring principle in light of research on pretense behavior in children. Here, I will focus on some other lines of psychological evidence. One such line of evidence concerns the way in which people engage in counterfactual thinking. Simply put, counterfactual thinking is what people do when they think about alternatives to reality. Imagination supports counterfactual thinking abilities, and when people think of alternatives to reality they tend to "create plausible alternatives to reality" (Byrne, 2007, p. 10). This is just what one would expect if principle (M) were in effect.

Further evidence in favor of $(\mathrm{M})$ can be found in the psychological literature on imagination and creative behavior. An early study supporting (M) is Ward (1994). Ward asked participants to imagine and draw alien animals. He found that participants tended to give creatures properties characteristic of the superordinate category: Animal. Moreover, participants tended to transfer attribute correlations from real animals to imaginary animals. For instance, if participants imagined an animal that 
had feathers, they also tended to give it wings. These and other findings led Ward to conclude that imagination processes were structured by participants' knowledge of applicable real world categories. ${ }^{3}$

A more recent finding directly supporting (M) is due to Weisberg and Goodstein (2009). Weisberg and Goodstein investigated how people fill in details that narratives leave unspecified. Armed with findings such as those of Ward (1994), they hypothesized that people will transfer facts from the real world to fictional worlds. They further hypothesized that the likelihood that a certain set of facts will get transferred depends on how different the fictional world is perceived to be from the real one, as well as on the type of fact in question (e.g., mathematical facts versus contingent facts).

To test their hypotheses, (Weisberg \& Goodstein, 2009) presented participants with three stories, each of which differed with respect to their similarity with reality. Upon reading the stories, paticipants were asked how likely they thought a set of 21 facts were to be true in the story worlds. Notably, the queried facts included twelve test facts that were never mentioned in the story. These twelve facts belonged to one of four fact types. Weisberg and Goodstein found a main effect of both distance to the real world and fact type, as predicted. They further found an interaction between the two factors - something unexpected. Note that, in addition to supporting (M), Weisberg and Goodstein's findings suggest paths for its refinement.

\footnotetext{
${ }^{3}$ It should be noted that Ward (1994) explicitly restricts his investigation to the "deliberate mental generation of a novel entity" (emphasis in original).
} 


\subsubsection{Why Mirroring Is Relevant}

I have presented psychological evidence that principle (M) is a plausible principle of generation. There are also theoretical considerations that render principle (M) salient to imagination systems research. Compliance with principle (M) stands to be a major factor in imagination system quality. Indeed, generation of imaginings in compliance with principle (M) appears to be one of the crucial functions of imagination systems.

Imagination is thought to support many cognitive abilities, among which are counterfactual thinking (Byrne, 2007), visuo-spatial thinking (Kosslyn, 1995), reasoning (Johnson-Laird, 1987) and metacognition (Currie \& Ravenscroft, 2002). It is thought that imagination supports these abilities in large part because it enables humans to simulate relevant possibilities (e.g., Currie \& Ravenscroft, 2002; Moulton \& Kosslyn, 2009). But for simulations to be informative, they have to correspond, at least to some extent, to the relevant states of affairs. For an embedded agent with limited resources and limited access to the world, principle (M) stands out as an efficient and effective way to ensure good simulation results.

As mentioned in the introductory chapter, artificial imagination systems are often implemented for applications that require the very same abilities imagination supports. Recall, for example, Ripley (Roy et al., 2004). Ripley performs a rudimentary form of metacognition by identifying and simulating relevant visual points of view in order to interpret natural language commands that contain spatial language. In other words, Ripley uses its ability to imagine to perform both a visuo-spatial thinking task and a metacognition task. Thus, it is likely that good mirroring performance stands to be a good predictor of overall performance for an imagination system. 


\subsection{MCA Automation}

Let us now consider how mirroring compliance can be operationalized. I will only consider the operationalization of the most salient features of mirroring. In particular, I will not address the concept of disparity, and I will not take into account psychological subtleties such as those discovered by Weisberg and Goodstein (2009). Furthermore, I will not address cases such as (8), where imaginers are asked to imagine unusual objects or to blend concepts.

These decisions are motivated by practical considerations. Compliance with mirroring is a complex phenomenon. We should first try to implement a simple, but credible approximation - one that assesses strict compliance with mirroring. Once such a method for assessing strict compliance with mirroring is developed, work can be done to extend it to accommodate disparity and other subtleties.

\subsubsection{Operationalization}

Recall principle (M).

(M) Elaborative choices follow from plausible inferences on imaginative prompts. An imagining can be construed as a set of elaborative choices on a given prompt. Therefore, to find an imagining plausible is to find the corresponding elaborative choices on the given prompt plausible. Likewise, to find an imagining implausible is to find the corresponding elaborative choices implausible. Therefore, compliance with (M) can be operationalized in terms of the plausibility of an imaginers' imaginings over some set of relevant prompts.

When we assess the plausibility of an imagining, we may assign it a scalar plausibility score, or we may assign it a categorical label. The score we assign reveals whether 
or not, or to what degree, an imagining is acceptable given our relevant background knowledge. In light of these observations, note the common sense observation that the more plausible we find something, the more likely we are to believe that it is true, or real. These observations taken together suggest the following operationalization: an imagining complies with mirroring to the extent that assessors are disposed to mistake it for ground truth data. In other words, a plausibility assessment can be modeled, to a first approximation, as a binary classification task.

\subsubsection{Mirroring and Binary Classification}

In binary classification, the goal is to determine whether some objects, represented as points in some feature space, belong to some relevant class. Generally, it is easy to obtain examples from the domain of interest, but difficult to formulate explicit classification rules. For this reason, classifiers are inferred from a set of labeled training examples. It is hoped that knowledge attained on the training set will generalize well over the entire domain of interest.

Binary classifiers can output both categorical decisions (accept or reject), and confidence scores. Training sets, feature sets and other aspects of classifier design can be chosen to represent prior knowledge required for a mirroring compliance assessment. Mirroring compliance can be measured as a value that varies negatively with classifier

performance. As a model of mirroring compliance assessment, binary classification meets all formal requirements.

\subsubsection{A Restriction On Training Data}

But now, consider a human mirroring compliance assessor's predicament in assessing whether or not a watermelon-sized banana-shaped blue apple is a mirroring compli- 
ant imagining for the prompt 'Imagine an apple'. The human assessor will likely have never been presented with such an apple, nor will the human have likely been presented with a large set of similar imaginings. That is to say humans make mirroring compliance judgments based on a very unbalanced set of knowledge: humans have extensive knowledge of the world but relatively little knowledge of the set of possible imaginings. This poses a challenge to the binary classification approach to mirroring compliance assessment.

The same predicament translates to mirroring compliance assessment for artificial imaginings. Suppose that we are interested in implementing a mirroring compliance assessor for some given domain $\mathcal{W}$. Training data must provide assessors with knowledge necessary to make decisions about the acceptability of arbitrary inputs. In general, these data may consist of acceptable or unacceptable exemplars. However, in order to implement valid mirroring compliance assessors, we must be reasonably sure that their judgments are unbiased. The set of unacceptable exemplars corresponds to the set of imaginings. Since our only access to imaginings is through previously implemented imagination systems, any sampling of imaginings for assessor training will be strongly biased. One possible way to get around this issue is to sample uniformly from the set of possible inputs, unfortunately this approach becomes unfeasible as the dimensionality of the input space increases (Tax, 2001). That is to say, artificial mirroring compliance assessors are in a similar predicament to human assessors.

Our predicament is that we are restricted to sampling training examples mostly (in the limit, only) from the set of acceptable inputs. This restriction presents a serious challenge to the binary classifier approach to mirroring compliance assessor implementation. In general, binary classifiers are trained by optimizing classifier per- 
formance. There are two factors that contribute to classifier performance. The first of these is classification correctness and the second is generalization. Classifier training aims to strike a good balance between classification correctness and generalization (Tax, 2001). When a training set lacks counterexamples or has very few of them, classifiers are not likely to generalize very well. This is because the lack of counterexamples means that little information is available for training algorithms to find an acceptable trade-off between classification correctness and generalization.

\subsubsection{MCA as a One-Class Classification Task}

We can accommodate the lack of counterexamples if we abandon the binary classification approach to mirroring compliance assessment in favor of a one-class classification approach. One-class classification and binary classification are closely related. Formally, both classification paradigms address the same problem, which can be expressed in probabilistic terms.

Let $\mathbf{x}$ be a feature vector sampled from a relevant feature space. Further, let $\omega_{T}$ indicate that $\mathbf{x}$ is an acceptable exemplar of $\mathcal{W}$ and $\omega_{O}$ denote that $\mathbf{x}$ is an unacceptable exemplar of $\mathcal{W}$. Both binary and one-class classifiers can be interpreted as estimators of the conditional probability of $w_{T}$ given the feature vector $\mathbf{x}, p\left(\omega_{T} \mid \mathbf{x}\right)$ (see Tax, 2001, for a more detailed treatment). ${ }^{4}$ In binary classification, $p\left(\omega_{T} \mid \mathbf{x}\right)$ can

be estimated directly. In one-class classification, this is not possible due to the lack of counterexamples (Tax, 2001). The remedy for this situation is analogous to sampling uniformly from the feature space, but it is much more efficient (Tax, 2001).

In one class classification, it is assumed that counterexamples are uniformly distributed around the data. Then, an application of Bayes' theorem allows us to es-

\footnotetext{
${ }^{4}$ I borrow Tax's (2001) notational conventions.
} 
timate the probability of $\omega_{T}$ given $\mathbf{x}, p\left(\omega_{T} \mid \mathbf{x}\right)$, with the probability of $\mathbf{x}$ given $\omega_{T}$, $p\left(\mathbf{x} \mid \omega_{T}\right)$. This is because, under the assumption that counterexamples are uniformly distributed around the data, $p\left(\omega_{T} \mid \mathbf{x}\right)$ is a monotone increasing function of $p\left(\mathbf{x} \mid \omega_{T}\right)$. To see why, consider an application of Bayes' theorem to $p\left(\omega_{T} \mid \mathbf{x}\right)$.

$$
p\left(\omega_{T} \mid \mathbf{x}\right)=\frac{p\left(\mathbf{x} \mid \omega_{T}\right) p\left(\omega_{T}\right)}{p(\mathbf{x})}=\frac{p\left(\mathbf{x} \mid \omega_{T}\right) p\left(\omega_{T}\right)}{p\left(\mathbf{x} \mid \omega_{T}\right) p\left(\omega_{T}\right)+p\left(\mathbf{x} \mid \omega_{O}\right) p\left(\omega_{O}\right)}
$$

To assume that counterexamples are uniformly distributed around the data is to assume that $p\left(\mathbf{x} \mid \omega_{O}\right)=c$, for some constant $c$. In contrast, in a binary classification setting, one would estimate $p\left(\mathbf{x} \mid \omega_{O}\right)$ using available counterexamples.

The assumption of uniformity changes the task from one of discriminating two sets of data to one of estimating a boundary around the data. Moreover, the problem of estimating $p\left(\mathbf{x} \mid \omega_{T}\right)$ is tractable with training data that only contains samples that satisfy $\omega_{T}$. To implement a one-class classifier, one infers an estimator of $p\left(\mathbf{x} \mid \omega_{T}\right)$ and sets a decision threshold $\theta_{d}$. An input $\mathbf{x}$ is then accepted if it is found that $p\left(\mathbf{x} \mid \omega_{T}\right)>\theta_{d}$ and rejected otherwise.

Like binary classification, one-class classification meets all formal requirements as a model of mirroring compliance assessment. Moreover, one-class classification is designed for scenarios in which counterexamples are not readily available. Mirroring compliance assessment is such a scenario. Therefore, strict mirroring compliance assessment can, in principle, be operationalized in terms of a one-class classification task on a domain of ground truth knowledge, $\mathcal{W}$, where compliance scores vary negatively with classifier performance. 


\subsection{Closing Remarks}

In this chapter I introduced the mirroring principle, which is a notable principle of generation. I then suggested that mirroring compliance assessment can be operationalized as a one-class classification task. In the next two chapters, I discuss an experiment in which I investigate the practical feasibility of automatic mirroring compliance assessment.

Before moving on, a few final comments are in order. In the previous chapter, I pointed out that imagination systems researchers' concern for implementing imagination systems with realistic outputs seemed to conflict with the proposition that imaginings cannot be evaluated according to their representational correctness (see Section 3.1.1). Imagination systems researchers' concern for realism can be interpreted as a desire to implement imagination systems with acceptable mirroring compliance. Interpreted this way, the paradox is resolved: evaluation is not based on whether or not imaginings are representationally accurate, it is based on whether or not they comply with the mirroring principle. The mirroring principle does not require imaginings to be representationally correct, only to be plausible to appreciators. 


\section{Chapter 5 Pilot MCA: Design and Methods}

The previous chapter introduced the mirroring principle, and it proposed an operationalization of mirroring compliance. In particular it was suggested that an imagining complies with mirroring to the extent that assessors are disposed to mistake it for ground truth data. This analysis yielded a concrete proposal for the implementation of automatic imagination system assessors: these assessors are to be implemented as one class classifiers tasked with detecting imaginings.

In the present chapter and the next, I present work done to implement an automatic mirroring compliance assessor in the domain of texture synthesis for visual media and to evaluate it against human assessors. This experiment pilots the mirroring compliance assessment methodology discussed in the previous chapter and investigates the practical feasibility of the proposal. The focus of the present chapter is methodological. I relate information concerning general experimental design, stimulus preparation, data collection and assessor implementation.

\subsection{Overview of Experiment}

From the proposal that an imagining complies with mirroring to the extent that assessors are disposed to mistake it for ground truth data, it follows that valid assessors should be more likely to mistake compliant imaginings for ground truth data. In 
order to test whether the construction of such assessors is feasible and fruitful, we need to establish what counts as compliant imaginings, but this is in general not an easy task. In imagination system applications where humans are the target audience, it is possible to collect judgments of mirroring compliance from human assessors.

In the present experiment I implemented a pilot mirroring compliance assessor for a specific kind of imagination task and I compared its behavior to qualified human assessors on the same domain using the same evaluation criteria. Specifically, the target task was to generate a visual texture for human media given a prompt denoting a texture category.

\subsubsection{Experimental Setting}

Visual textures are visual patterns that "are spatially homogeneous and consist of repeated elements, often subject to some randomization in their location, size, color, orientation, etc." (Portilla \& Simoncelli, 2000). Visual textures are often the consequence of the surface structure of materials, and are consequently useful for identifying and segmenting materials. Moreover, visual textures are parts of the natural world with which ordinary human beings are closely acquainted and, consequently, they are an important part of the visual world.

It is no surprise, then, that texture synthesis has many applications in media such as games and art. But, human familiarity with visual textures also means that texture synthesis is a convenient domain of imagination systems research for piloting automatic mirroring compliance assessors. Notably, there are at least five reasons to choose texture synthesis for visual media as the target task for such a pilot project. First, fairly large texture datasets are readily available, making assessor training relatively easy. Second, there are several powerful texture synthesis algorithms available, 
which ensures that the task will not be trivial. Third, the high dimensionality of visual images adds further challenge to the task. Fourth, it has been found that humans are sensitive to artifacts that texture synthesis algorithms introduce into synthesized images (Liu, Dong, Cai, Qi, \& Chantler, 2015). Moreover, Balas (2006) has found that humans are sensitive to the presence or absence of statistical image features used by a state-of-the art texture synthesis model in that their discrimination performance varies with the presence or absence of these statistical features. These findings suggest that humans should fare well in a mirroring compliance assessment task where they must identify synthesized textures. Finally, since texture discrimination is a relatively effortless perceptual task, it is easy to collect data from human assessors for validation purposes.

\subsubsection{Methodology}

In domains where evaluation criteria are complex or ill-defined, human assessors are evaluated with respect to their agreement with peers. In such domains, it is assumed that a candidate's average score over all possible test administrations is their true score on the task, and good assessors are considered to be assessors that are representative of average assessor behavior (Page, 1994). Consequently, in a group of qualified assessors, the assessor with the highest overall pairwise agreement with their peers is considered to be the highest performing assessor.

Pairwise agreement between human assessors and machine assessors has been used with some success for automatic assessor validation research in other domains (e.g., Page, 1994, for essay grading). In such an experimental paradigm, both human and machine assessors are held to the same standard of evaluation. The desired result is that the pairwise average agreement between human and machine assessors be at 
least as large as the pairwise average agreement among human assessors. For the present experiment, I adopted this approach to assessor evaluation as it put human and machine assessors on an equal footing and as it did not knowledge specific to texture perception.

In particular, I investigated how pairwise agreement for human-machine (H-M) and human-human $(\mathrm{H}-\mathrm{H})$ pairs agreed in the rankings and scores they assigned to three texture synthesis systems relative to each other and relative to a ground truth data set. Assessors were shown images along with corresponding imaginative prompts (independent variables, including assessors) and asked to assign a score to each image (dependent variable). These scores were then used to compare pairwise agreement for $\mathrm{H}-\mathrm{M}$ and $\mathrm{H}-\mathrm{H}$ pairs.

The indicators of agreement used in this experiment are, the generalizability coefficient $\left(\mathbf{E} \rho^{2}\right)$ and the index of dependability $(\boldsymbol{\Phi})$. Both of these statistics are defined within the framework of generalizability theory, a theory dedicated to investigating the reliability of measurements. However, the two statistics have different interpretations. The generalizability coefficient is, roughly, a measure of agreement in terms of the relative ranking of objects of measurement. The index of dependability, on the other hand, is a measure of agreement in terms of the absolute score awarded to objects of measurement. Procedural details for computing $\mathbf{E} \rho^{2}$ and $\boldsymbol{\Phi}$ are available in Section 6.1.

\subsection{Experimental Stimuli}

A total of 220 grayscale images were used as stimuli. Images were sampled from 4 sources and 11 prompts. Image sources were either the TIPS-2b texture image dataset (Mallikarjuna et al., 2006) or one of three texture synthesizing imagination 
systems. Image prompts corresponded to the 11 texture categories represented in the TIPS-2b dataset (Table 5.1). Each source-prompt pair was presented on five distinct occasions; for analysis, data from these occasions were aggregated by sourceprompt pair and normalized. Test images were presented along with their prompts. For human assessors, the prompt was indicated by text placed next to the image (see Figure 5.2); for artificial assessors the prompt was formatted as a one-hot vector and presented as input along with a representation of the image (see Section 5.4.1). Images from the TIPS-2b dataset also served as training data for the artificial assessor (see 5.4.2).

The texture synthesizing imagination systems were based on the Portilla-Simoncelli texture model (Portilla \& Simoncelli, 2000). For the experiment to work, it was crucial to generate a set of systems that would reliably differ in the quality of the textures they synthesized. The Portilla-Simoncelli model, which is the current state-of-the art in parametric texture synthesis (Rosenholtz, 2014), greatly facilitated this process. The model operates according to the "synthesis by analysis" approach. Local image statistics are collected for a texture of interest and, in the synthesis step, the synthesized image is iteratively modified to converge to these statistics. Moreover, the model allows for the inclusion or exclusion of different sets of image statistics in the synthesis process, making it ideal for generating texture synthesizers of differing quality as it is possible to 'lesion' the system in a way so as to promote differences in performance.

Though support for color images was added to an updated version of the PortillaSimoncelli model, ${ }^{5}$ I opted to use implement the experiment using grayscale im-

\footnotetext{
${ }^{5}$ Color-compatible version of the Portilla-Simoncelli texture synthesis algorithm is available at http://www.cns.nyu.edu/ lcv/texture/
} 
Table 5.1

Texture Categories Represented in the TIPS-2b Dataset

\begin{tabular}{l}
\hline Aluminium Foil \\
Brown Bread \\
Corduroy \\
Cork \\
Cotton \\
Cracker \\
Lettuce Leaf \\
Linen \\
White Bread \\
Wood \\
Wool \\
\hline
\end{tabular}

ages. Previous research studying the perceptual characteristics of the model use the grayscale version (Balas, 2006). Furthermore, use of color images would have significantly increased computational demands associated with training the artificial assessor.

\subsubsection{TIPS-2b Images}

Work on texture perception (e.g., Balas, 2006) that relies on natural texture images often makes use of relatively small sets of texture images. In contrast, to implement and validate a mirroring compliance assessor for natural texture synthesizers, we need a relatively large dataset that is representative of the variety and variability found in natural textures. The TIPS-2b dataset contains $200 \times 200$ RGB images sampled from 4 items from each of 11 texture categories that are imaged from a variety of orientations and distances (Mallikarjuna et al., 2006). The size of the dataset as well as the systematicity of its image collection methodology make it an ideal dataset for our current purposes. For these reasons, it was used as a source of ground truth 
natural texture images.

\subsubsection{Image Preprocessing}

TIPS-2b images were converted to grayscale and cropped around their center to be of size $128 \times 128$ to be compatible with the implementation of the Portilla-Simoncelli texture model (Portilla \& Simoncelli, 2000) that was used. Some TIPS-2b images do not have the $200 \times 200$ dimensions. Often images fail to have $200 \times 200$ dimensions because at larger imaging distances, the subject material does not "fill a sufficiently large part of the image" (Mallikarjuna et al., 2006) (e.g., a cracker imaged from about $64 \mathrm{~cm}$ away). Variability in the image dimensions would have significantly complicated the design of artificial assessors. Therefore, only images with dimensions $200 \times 200$ were used.

\subsubsection{Stimulus Preparation}

After preprocessing, a set of 4519 images were available for use. These images were split into two sets. Two-hundred and twenty (220) images were randomly chosen to construct the stimulus set. The remainder of the images were used as training data for the artificial assessor. Of the 220 images chosen to consturct the stimulus set, 55 (5 from each texture category) were set aside as ground truth images, to be presented to assessors unaltered. The remaining 165 images were used to synthesize texture images using the Portilla-Simoncelli texture model (Portilla \& Simoncelli, 2000). The NYU Laboratory for Computational Vision implementation of the model was used during texture synthesis. ${ }^{6}$ Sample images from the test set can be seen in Figure 5.1.

The Portilla and Simoncelli (2000) texture model uses (i) marginal image statis-

\footnotetext{
${ }^{6}$ Available at http://www.cns.nyu.edu/ lcv/texture/
} 


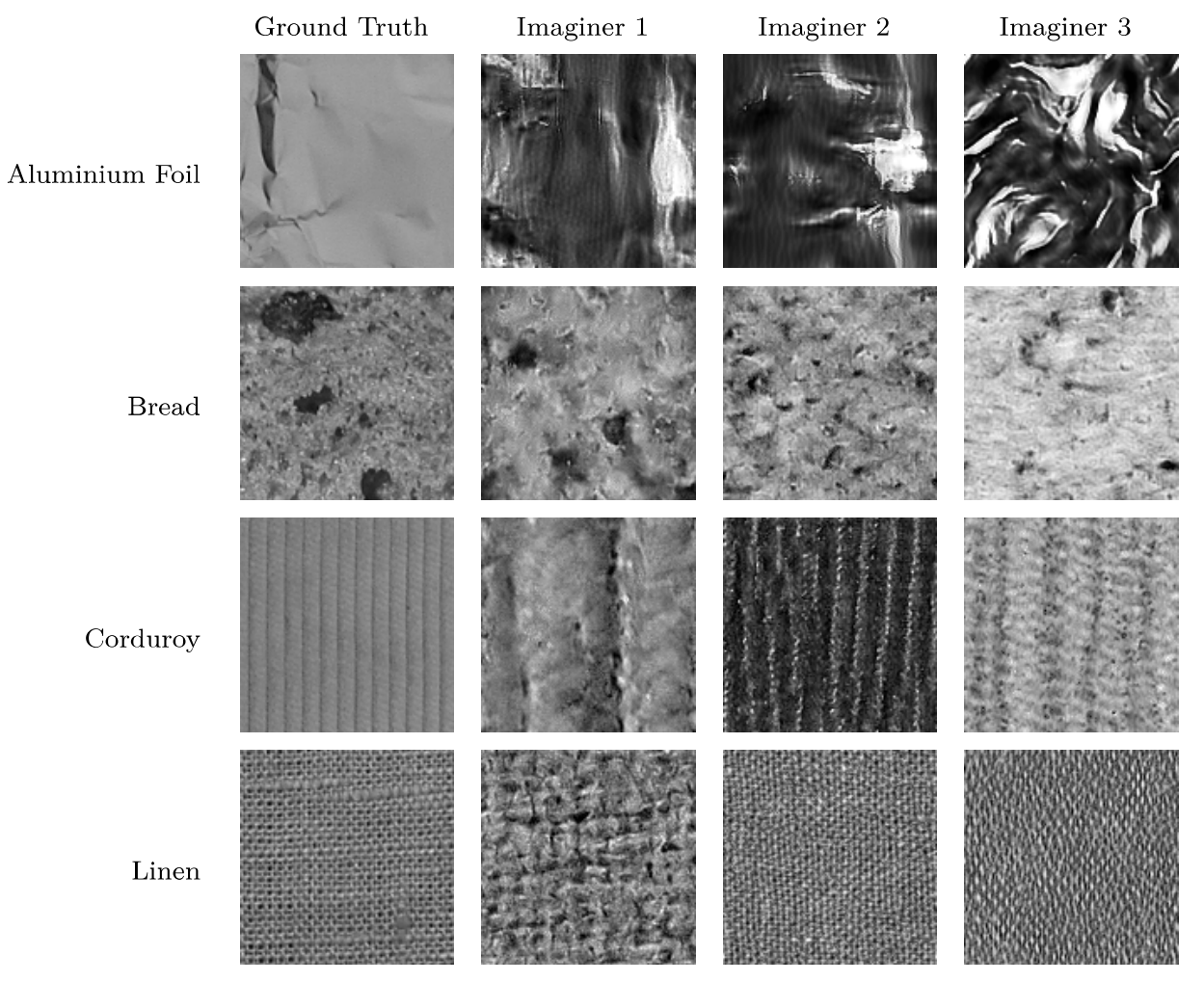

Figure 5.1. Sample Images from the Test Set. 
tics, (ii) coefficient correlations, (iii) magnitude correlations and (iv) cross-scale phase statistics for texture synthesis and analysis. For the present experiment, three imaginers were created by a process of progressive lesioning. Imaginer $1\left(\mathfrak{I}_{1}\right)$ was implemented using the full parameter set. Imaginer $2\left(\mathfrak{I}_{2}\right)$ omitted cross-scale phase statistics in the texture synthesis step. Finally, Imaginer $3\left(\mathfrak{I}_{3}\right)$ omitted both crossscale phases statistics and coefficient correlations during the synthesis step. For the

remaining parameters I used settings reported in the original Portilla and Simoncelli (2000) paper ( $N=4, K=4, M=7$ with 50 iterations). The remaining images selected to be part of the test set were partitioned into balanced sets of 55 images and used to generate 55 imaginings using each of $\mathfrak{I}_{1}, \mathfrak{I}_{2}$, and $\mathfrak{I}_{3}$.

\subsection{Human Assessor Data Collection}

Participants were presented with a forced choice task where they had to either judge an image to be an imagining or a photograph. Methods employed for human assessor data collection are summarized below.

\subsubsection{Participants}

Twenty nine (29) participants were recruited on the Crowdflower crowdsourcing service as image assessors. Participants gave online consent for the collection of their data (CUREB Project \#104628). Assessors self-reported as having normal or corrected to normal vision; they were compensated for their time with a small monetary gift. One assessor was found to be an outlier upon visual inspection of their data and was excluded from analyses. 


\subsubsection{Stimuli and Materials}

Experimental stimuli were the test images described in Section 5.2. There was a total of 220 images. Additionally, a briefing text describing key study concepts and a set of three multiple choice comprehension questions were presented to assessors to ensure that they understood task requirements. The briefing text and comprehension questions are reproduced in Appendix A.

\subsubsection{Design}

A balanced and fully crossed design was employed. For each assessor ( $a=28$, outlier excluded), five judgements were collected for every combination of image source $(s$, $\left.n_{s}=4\right)$ and prompt $\left(p, n_{p}=11\right)$ to yield a total of $4 \times 11 \times 28 \times 5=6160$ judgements.

\subsubsection{Procedure}

Assessors completed the experiment online, on an interface implemented using the LimeSurvey survey software. At the start of the experiment, some text explaining the task and key concepts, such as Natural Texture, Imagination System and ImAGINING, was presented. Assessors were allowed to study this text at their leisure. See Appendix A for the text that briefed participants on key concepts.

Upon completing study of experimental concepts and procedure, assessors were presented with comprehension questions (also available in Appendix A); they could attempt these questions as many times as they liked, but could not advance if they did not give the correct answer. Once assessors answered all comprehension questions correctly, they moved on to the main phase of the experiment.

In each trial of the main phase, assessors were shown the stimulus image along with the corresponding prompt and asked to indicate if they thought the image was a 
Is the following visual texture image a computer generated imagining or a photograph?

Aluminium Foil

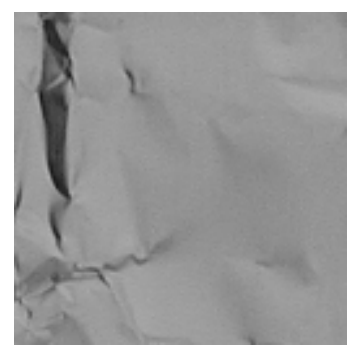

Figure 5.2. Reproduction of an Experimental Trial.

photograph or an imagining. Each stimulus image was presented exactly once and in random order. Assessors could respond by clicking an on-screen button or by using the arrow keys on their keyboard.

\subsection{Assessor Implementation}

A common design pattern for texture processing systems is to use a linear filter followed by a non-linear filter followed, again, by a linear filter (Pappas, Neuhoff, De Ridder, \& Zujovic, 2013). It has been found that this design supports good performance in texture discrimination. Replicator neural networks (RNNs) can comply with the linear-nonlinear-linear architecture when they have multiple non-linear hidden layers (Tax, 2001; Japkowicz, Jos, \& Gluck, 2000). Consequently, the artificial assessor investigated in this experiment was built around an RNN with three nonlinear hidden layers.

RNNs are neural networks that are trained to reproduce their input at their output (Hawkins, He, Williams, \& Baxter, 2002). Usually, an information bottleneck is 


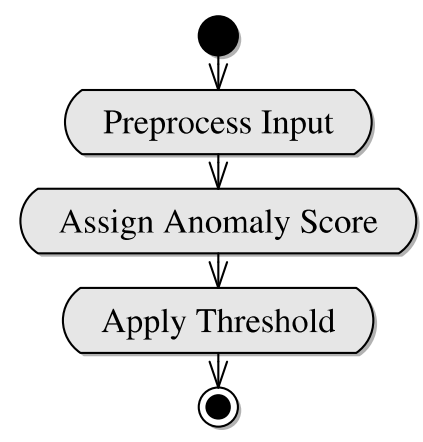

Figure 5.3. High-level View of Automatic Assessor Behavior.

implemented to encourage the network to learn useful representations of the data. In a one-class classification setting, one assumes that unusual exemplars will prove more difficult for an RNN to reconstruct than more ordinary exemplars. The reproduction error is interpreted as an anomaly score (Hawkins et al., 2002; Tax, 2001). Therefore, a threshold on reproduction error can serve as an effective decision threshold for the classifier.

\subsubsection{Design}

The general architecture of the artifcial assessor can be seen in Figure 5.3. Input to the mirroring compliance assessor consisted of $128 \times 128$ grayscale pixel images along with a label for representing the texture category (imaginative prompt). The mirroring compliance assessor processed data in three steps. In the first step, data was preprocessed for dimensionality reduction and formatting purposes. In the second step, an RNN was used to obtain reproduction errors. RNN design is detailed in Figure 5.4. Finally, a simple quantile estimator was used to implement a thresholding mechanism.

Image data was scaled to have zero mean and data dimensionality was reduced us- 


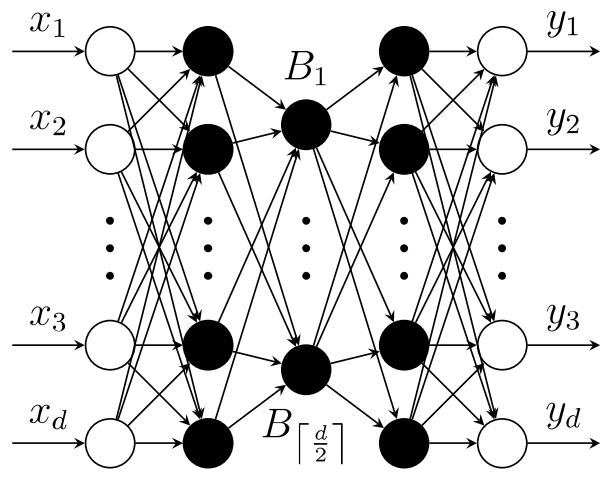

Figure 5.4. Network architecture. Input values are shown as $x_{i}$, output values as $y_{i}$. $B_{i}$ designates units in the bottleneck layer. Sigmoid units are shown in black, linear units in white. The network had a feed-forward design with 3 hidden layers. Hidden layers other than the bottleneck layer contained $d$ units each, where $d$ designates the number of input dimensions.

ing principal components analysis. In applications where data has high-dimensionality and also high redundancy, such as visual texture discrimination, principal components analysis is an effective tool to scale down data dimensionality while preserving important variation in the data. Texture labels were encoded as one-hot vectors and concatenated with the dimensionality-reduced data vector representing the texture image before being presented to the RNN.

RNNs can readily be used to obtain anomaly scores for data points. However, to my knowledge, previous work on RNN based anomaly detection does not explicitly incorporate a tunable decision threshold $(\theta)$. Instead, decision thresholds are either omitted (Hawkins et al., 2002), or determined heuristically (Dau, Ciesielski, \& Song, 2014). Here, it was necessary to implement a tunable decision threshold in order to obtain adequate results. In one-class classification, the decision threshold is often 
arbitrarily chosen. However, for the present task a meaningful choice of the threshold parameter was available (see 5.4.2).

Recall that one-class classifiers estimate the probability, $p\left(\omega_{T} \mid \mathbf{x}\right)$, that an input is acceptable using the probability, $p\left(\mathbf{x} \mid \omega_{T}\right)$, of the input assuming that it is acceptable. For RNNs, higher reproduction errors are expected to correlate with higher likelihood that the input is an anomaly. This is to say that the probability $p\left(\mathbf{x} \mid \omega_{T}\right)$ can be estimated in terms of the reproduction error on $\mathbf{x}\left(\varepsilon_{\mathbf{x}}\right)$.

Since reproduction errors on acceptable inputs are expected to be low, $p\left(\mathbf{x} \mid \omega_{T}\right)$ can be defined as the complement of the quantile probability estimate of $\varepsilon_{\mathbf{x}}$, when it is assumed that $\mathbf{x}$ is an acceptable input, as in (9).

(9) $p\left(\mathbf{x} \mid \omega_{T}\right):=1-p\left(\varepsilon<\varepsilon_{\mathbf{x}} \mid \omega_{T}\right)$

A reproduction error threshold can then be determined by finding an estimate, $\hat{\varepsilon}_{\theta}$, for $\varepsilon_{\theta}$, where $\varepsilon_{\theta}$ satisfies $(10)$.

$$
\theta=1-p\left(\varepsilon<\varepsilon_{\theta} \mid \omega_{T}\right)
$$

A subset, $\mathcal{S}$ of the training data was used to determine the estimate $\hat{\varepsilon}_{\theta}$. The order statistic $S_{(k)}$ was computed for $S_{1}, \ldots, S_{|\mathcal{S}|}$ in $\mathcal{S}$, where $k$ is defined by (11) (see Davis \& Steinberg, 2006).

(11) $k= \begin{cases}|\mathcal{S}| \cdot \theta & \text { if }|\mathcal{S}| \cdot \theta \text { is an integer } \\ \lfloor|\mathcal{S}| \cdot \theta\rfloor+1 & \text { otherwise. }\end{cases}$

Then, $\hat{\varepsilon}_{\theta}$ was set such that $\hat{\varepsilon}_{\theta}=S_{(k)}$.

\subsubsection{Training Procedure}

Images that did not get placed in the test set were further randomly split into three subsets. These subsets included two training sets $\left(\mathcal{X}_{1}\right.$ and $\mathcal{X}_{2}$, containing 2579 and 
1290 images respectively) and a validation (430 images) set.

Training set $\mathcal{X}_{1}$ was used to fit the preprocessors and to train the RNN, whereas training set $\mathcal{X}_{2}$ was used to determine the reproduction error threshold. Splitting the training set into two subsets in this way was adopted after it was found that the network performed poorly on validation data. I attributed this to the threshold quantile estimate being biased due to data reuse. Upon splitting the dataset, performance was noticeably better (the assessor rejected the desired proportion of ground truth data) even though a significantly smaller ground truth data sample was being used to set the threshold.

Dimensionality reduction was performed to render the learning procedure tractable in terms of computational and temporal resources. At least $90 \%$ of variance was kept on $\mathcal{X}_{1}$, this reduced the original $128 \times 128$ image vector to a 724 -dimensional dimensionality-reduced image vector. The dimensionality-reduced image vectors were presented to the RNN along with one-hot vectors representing image prompts. Thus, input to the RNN had $d=735$ dimensions. The RNN was trained using backpropagation with momentum $\left(r_{\text {learn }}=.001, m=0.9\right)$. Training parameters were determined by manual search.

For training and for quantile estimation purposes, network error was computed according to (12).

$$
\varepsilon_{\mathbf{x}}=\frac{1}{d} \sum_{i=1}^{d} w_{i}\left(y_{i}-x_{i}\right)^{2}
$$

The variables $x_{i}, y_{i}$ denote network inputs and outputs as shown in Figure 5.4. Weigths $w_{i}$ represented the importance of the error term on the $i$ th input value. For image data, this weight was proportional to the variance explained by the corresponding principal component. For the one-hot prompt vectors, all error components 
were evenly weighted. The weights were scaled so as to give an even weighting to error on image data and on prompt data and so that they summed to 1 overall.

Training set $\mathcal{X}_{2}$ was used to obtain the reproduction error threshold. The threshold $\theta$ was chosen to match the rate at which human raters rejected ground truth images. This rate can be read from Figure 6.1.

\subsubsection{Running the Test Images}

All 220 stimulus images described in Section 5.2 were presented to the automatic

assessor, just as was done to the human assessors. Just as human assessors had access to the imaginative prompts associated with the test images, the assessor also had access to this information in the form of one hot vectors. Responses were recorded and aggregated as described in Section 5.2.

\subsection{Closing Remarks}

The present chapter introduced an experiment designed to pilot the mirroring compliance assessment methodology discussed in Chapter 4 and to investigate the practical feasibility of the proposal. So far, we have discussed procedural details of the experiment. The next chapter contains an analysis of the data obtained from the experiment and a discussion of the results. 


\section{Chapter 6 Pilot MCA: Results and Discussion}

In the previous chapter I described the design and methodology for an experiment dedicated to comparing an artificial mirroring compliance assessor to human mirroring

compliance assessors. Both artificial and human assessors were presented with natural texture images for mirroring compliance assessment. Notably, images were sourced either from a ground truth dataset $(\mathbb{T})$ or generated by three imaginers, $\mathfrak{I}_{1}, \mathfrak{I}_{2}$ and $\mathfrak{I}_{3}$ of differing quality.

The goal of the experiment was to investigate the extent to which the artificial assessor will agree with qualified human assessors on the mirroring compliance assessment task in question. In this chapter, I present the results of this experiment along with some discussion. Recall that the general strategy of the experiment is to compare agreement scores for human-machine (H-M) pairs to agreement scores for human-human $(\mathrm{H}-\mathrm{H})$ pairs. I start the chapter with a detailed description of the procedures for calculating agreement scores. I then present the results and I close with the discussion.

\subsection{Overview of Generalizability Theory and Agreement Statistics}

Generalizability theory ( $\mathrm{G}$ theory) is a framework designed to assess "the dependability of behavioral measurements" (Shavelson \& Webb, 1991). The dependability of a 
test is the extent to which scores on a single administration of the test yields results that reflect the average score a subject would receive under all possible acceptable alternative administrations of the same test. In $\mathrm{G}$ theory, one defines a universe of admissible observations, the set of all possible conditions under which a score assignment can be made, and partitions it into objects of measurement and facets. Facets are sources of variability in assigned scores that contribute to measurement error whereas objects of measurement are sources of variability that are of interest to testers. For example, for a multiple choice test, one may distinguish two sources of score variation: students and test items. If one is interested in evaluating student performance, students are the object of measurement. Test items, on the other hand, are a facet: they introduce sources of variation that do not reflect student ability (e.g. score variation due to item difficulty or arbitrary interactions between items and student performance).

G theory allows one to assess the contributions of the object of measurement and facets to overall score variation (Shavelson \& Webb, 1991). This information can then be used to assess the reliability of various test designs and to optimize testing procedures. First variance components are estimated using techniques such as ANOVA, this stage is called a generalizability $(G)$ study. The variance components obtained from a G study can be used to estimate the variance components in test designs of interest. These estimated components can then be used to assess the reliability of designs of interest with the help of two coefficients: the generalizability coefficient $\left(\mathbf{E} \rho^{2}\right)$ and the index of dependability $(\mathbf{\Phi})$. The choice of coefficient depends on the kinds of decision the test is designed to support. For relative decisions, such as rank ordering candidates in a competition, the generalizability coefficient should 
be used. For absolute decisions, such as failing a student if they receive less than $\% 50$ in a course, the dependability coefficient should be used. These additional steps are called decision studies. For an in-depth treatment of G theory, see Brennan (2001).

\subsubsection{Computing $\mathbf{E} \rho^{2}$ and $\Phi$}

The generalizability coefficient $\left(\mathbf{E} \rho^{2}\right)$ and the index of dependability $(\mathbf{\Phi})$ can be interpreted as agreement measures (Brennan, 2003). E $\rho^{2}$ measures agreement with respect to relative rankings of objects of measurement, whereas $\boldsymbol{\Phi}$ measures absoulte scores awarded to objects of measurement. These coefficients are related to Cron-

bach's $\alpha$, but wheras Cronbach's $\alpha$ is only applicable in the presence of one facet of measurement error, the $\mathrm{G}$ theory coefficients are applicable, in theory, when there are an arbitrary number of facets of measurement error (Brennan, 2003).

For the present experiment we need only be concerned with what are known as random fully crossed $\mathrm{G}$ study designs. In a fully crossed design, measurements are available under every possible condition of measurement for the object of measurement and for all facets of error. By way of example, consider the data collected for the present experiment. For the present experiment the object of measurement is the image source $(s)$, as we are interested in how assessors assign mirroring compliance scores to the three imaginers that generated the test images. The facets of error are prompts $(p)$ and assessors $(a)$. This is because there may, for example, be variability in how strict assessors are with respect to accepting an image as mirroring-compliant. There may also be interactions between different conditions of measurement. Take note that, in our example there are $n_{s}=4$ sources, $n_{p}=11$ prompts, and $n_{a}=28$ human assessors.

As mentioned above, the first step in computing $G$ theory related coefficents is 
to conduct a G Study - that is, to estimate variance components for every possible source of variance in the design. For the fully crossed design (represented as $s \times p \times a$ ) described above, there are seven variance component-one for effects associated with each object of measurement or facet of error and one for each possible interaction. Let us mark these variance components using subscripts to denote effects and interactions.

$$
\sigma_{s}^{2}, \sigma_{p}^{2}, \sigma_{a}^{2}, \sigma_{s p}^{2}, \sigma_{s a}^{2}, \sigma_{a p}^{2}, \sigma_{s a p, e}^{2}
$$

These variance components represent variance for individual judgments, and not for the overall score assigned to an imaginer.

When we are interested in assigning a mirroring compliance score to an imaginer, we take into consideration not single judgments, but an average of judgments. As we average judgments under different conditions of measurement, we begin to eliminate measurement error. We can use the variance components obtained in our G study to estimate the reliability of average judgments obtained in an assessment procedure of arbitrary design, with arbitrary numbers of measurements under each condition of measurement. This estimation process consists in a decision study. For our purposes, we need only be concerned with the case where the design of the decision study is identical to the design of the G study.

In order to compute $\mathbf{E} \rho^{2}$ and $\Phi$, we must first compute relative and absolute error. In a decision study where the design is identical to the corresponding G study, relative error is defined thus as follows:

$$
\sigma_{\delta}^{2}=\frac{\sigma_{s p}^{2}}{n_{p}}+\frac{\sigma_{s a}^{2}}{n_{a}}+\frac{\sigma_{s a p, e}^{2}}{n_{p} n_{a}}
$$


On the other hand, absolute error is computed as follows:

$$
\sigma_{\Delta}^{2}=\frac{\sigma_{p}^{2}}{n_{p}}+\frac{\sigma_{a}^{2}}{n_{a}}+\frac{\sigma_{s p}^{2}}{n_{p}}+\frac{\sigma_{s a}^{2}}{n_{a}}+\frac{\sigma_{a p}^{2}}{n_{p} n_{a}}+\frac{\sigma_{s a p, e}^{2}}{n_{p} n_{a}}
$$

Only variance components for interactions involving the object of measurement $(s$ in this case) contribute to relative error whereas variance components for all effects and interactions that are not due to the object of measurement contribute to absolute error. In both computations, each variance component contributing to error is divided by the product of the number of conditions under each facet associated with that variance component. For random fully crossed decision studies incorporating an identical design with fewer or more numerous facets of error, the computations are the same, adjusted for the number of facets of error.

Finally, when one has computed relative and absolute measurement errors as described above, one can compute coefficents $\mathbf{E} \rho^{2}$ and $\Phi$ for object of measurement $s$ according to the formulas below.

$$
\mathbf{E} \rho^{2}=\frac{\sigma_{s}^{2}}{\sigma_{s}^{2}+\sigma_{\delta}^{2}} \quad \Phi=\frac{\sigma_{s}^{2}}{\sigma_{s}^{2}+\sigma_{\Delta}^{2}}
$$

For the present analyses, we will only compute $\mathbf{E} \rho^{2}$ and $\Phi$ with image source $(s)$ as object of measurement

\subsection{Results}

Data collected from human assessors and generated by the artificial assessor were analyzed in two steps. First, human assessors' image acceptance rates were examined to evaluate the validity of human assessors' judgments for the mirroring compliance as- 


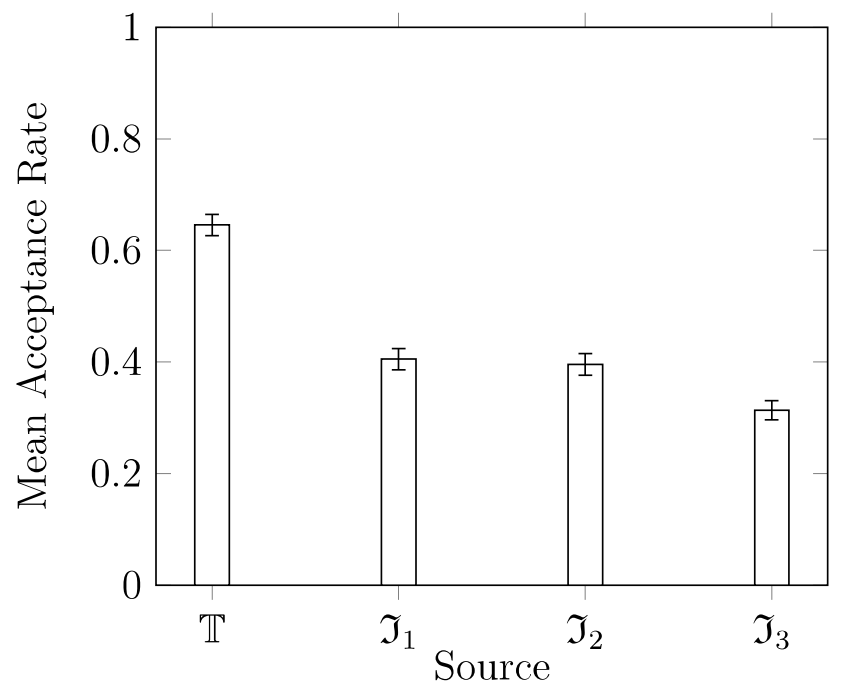

Figure 6.1. Image acceptance rates for human assessors by image source. Error bars indicate standard errors.

sessment task. Then, pairwise comparisons were conducted between human-machine (H-M) and human-human (H-H) pairs in order to investigate the performance of the artificial asssessor.

\subsubsection{Analysis of Human Judgments}

Human assessors' image acceptance rates can shed light on the difficulty of the mirroring compliance assessment task. Human image acceptance rates broken down by image source are displayed in Figure 6.1. Inspection of Figure 6.1 suggests significant differences in acceptance rates between each pair of sources, except for the pair $\mathfrak{I}_{1}$, $\mathfrak{I}_{2}$. Human assessors are able to discriminate imaginers from ground truth data, and $\mathfrak{I}_{3}$ appears to perform worse than the other two imaginers.

Figure 6.2 displays human image acceptance rates for ground truth images broken down by image prompt. Acceptance rates in the figure are ordered from smallest to 


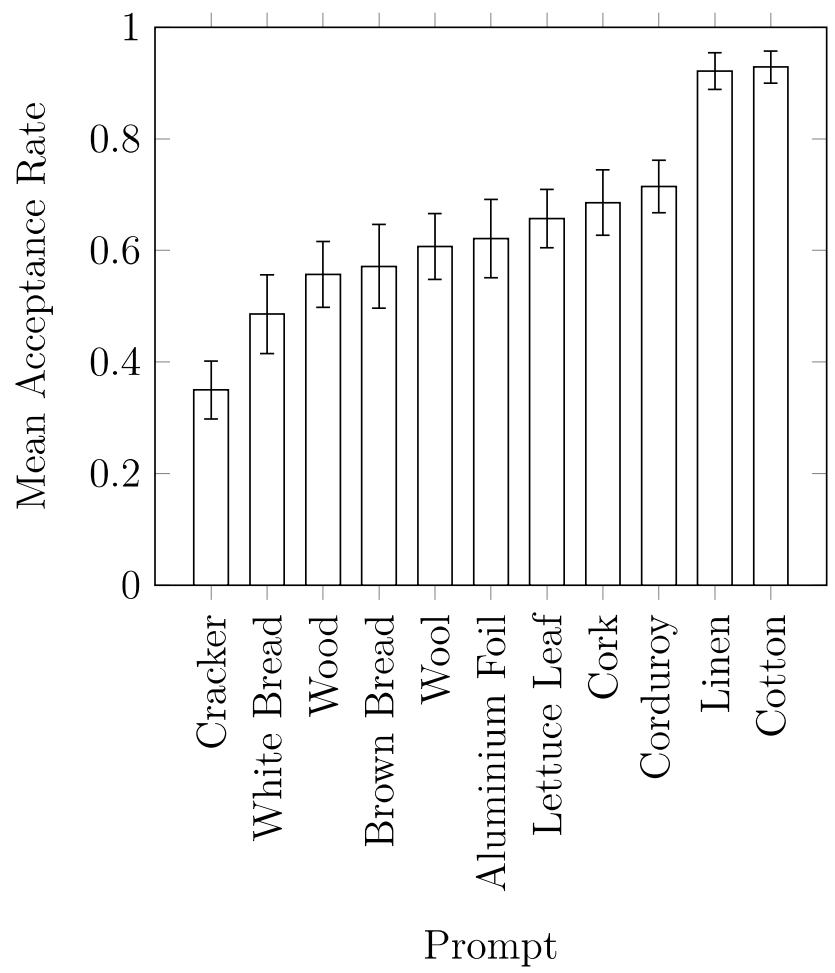

Figure 6.2. Human Image Acceptance Rates by Prompt. Error bars indicate standard errors.

largest to facilitate reading. Low acceptance rates on ground truth images for some prompts suggests that the images associated with these prompts were unfamiliar to human assessors and thus of questionable ecological validity.

In conclusion, human assessors were able to discriminate imaginers from ground truth and they were even able to discriminate between imaginers, in the case of $\mathfrak{I}_{3}$. However, some prompts may have low validity due to low acceptance rates for associated ground truth images. 


\subsubsection{Comparison of Human-Machine and Human-Human Pairwise Agreement Scores}

In order to compare $\mathrm{H}-\mathrm{M}$ and $\mathrm{H}-\mathrm{H}$ pairs, individual fully crossed random $\mathrm{G}$ and decision studies were conducted for each pair. In these studies, the conditions of measurement included data source $\left(s, n_{s}=4\right)$, imaginative prompt $\left(p, n_{p}=11\right)$, and assessor $\left(a, n_{a}=2\right)$.

Agreement scores for every possible $\mathrm{H}-\mathrm{M}$ and $\mathrm{H}-\mathrm{H}$ pair was calculated using the procedure described in Section 6.1.1. Initially, there were a total of 28 human-machine pairs and 378 human-human pairs. However, it was found that two human assessors consistently had 0 agreement when paired with other human assessors. If included, these participants biased results in favor of the artificial assessor. Consequently, they were excluded from analysis. Upon exclusion of these participants, there were $26 \mathrm{H}-$ $\mathrm{M}$ and $325 \mathrm{H}-\mathrm{H}$ pairs respectively. Descriptive statistics of agreement scores by pair type with respect to both relative and absolute measures of agreement are displayed in Figure 6.3.

Figure 6.3 reveals that mean relative agreement among $\mathrm{H}-\mathrm{H}$ pairs is slightly above mean agreement among H-M pairs. Nevertheless, human-machine agreement seems to be near human-human agreement levels for relative decisions. Agreement scores are notably lower for the index of dependability for both kinds of pairings. This is to be expected due to the way the two agreement scores are calculated. In terms of absolute agreement scores, H-M pairs exhibit greater mean agreement than humanhuman pairs. Moreover, differences between mean agreement scores for the index of dependability are more pronounced compared to the differences in mean agreement scores as measured by the generalizability coefficient. H-M agreement for absolute decisions seems to be at, or even perhaps above, human-human levels, though, overall, 


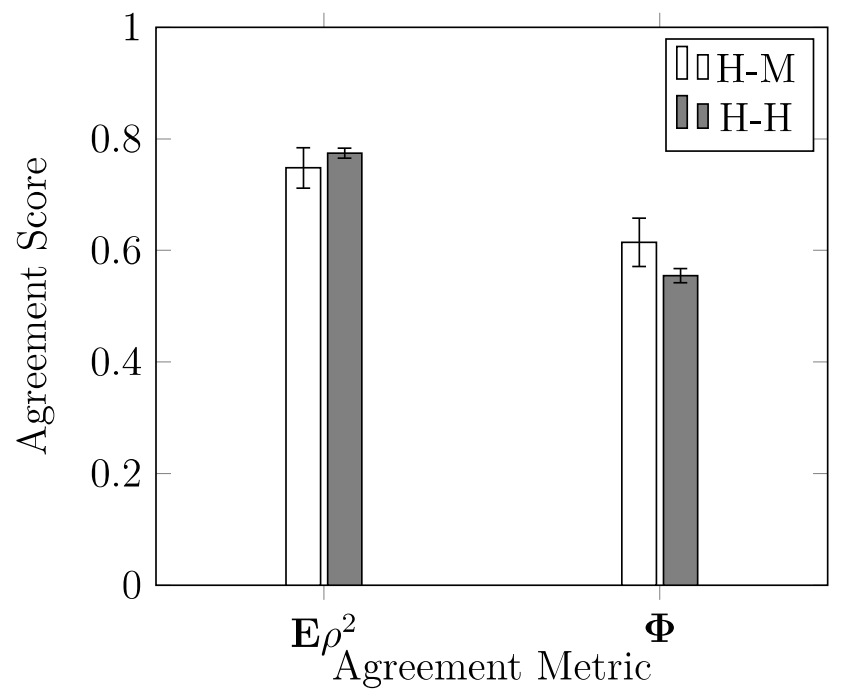

Figure 6.3. Mean human-machine (H-M) and human-human $(\mathrm{H}-\mathrm{H})$ pairwise agreement scores for both agreement metrics. Error bars indicate standard errors.

pairwise agreement scores for absolute decisions remain questionable. However, it is worth taking note of the fact that agreement score values are not due to chance agreement, as when the procedure is run with random assessors, agreement scores are equal to zero.

In order to investigate how the artificial assessor behaved in contrast with human assessors, H-M and H-H pair data was further split by imaginative prompt. Then G and decision studies similar to those presented above were conducted. In this case, the conditions of measurement included only data source $\left(s, n_{s}=4\right)$ and assessor ( $a$, $\left.n_{a}=2\right)$. The study design remained fully crossed and random.

Figures 6.4 and 6.5 display mean pairwise agreement for H-M and H-H pairs for each imaginative prompt for the generalizability coefficient and the index of dependability respectively. In both figures, data are ordered according to $\mathrm{H}-\mathrm{H}$ pairwise agreement. This allows for comparing H-M performance to $\mathrm{H}-\mathrm{H}$ performance on a 


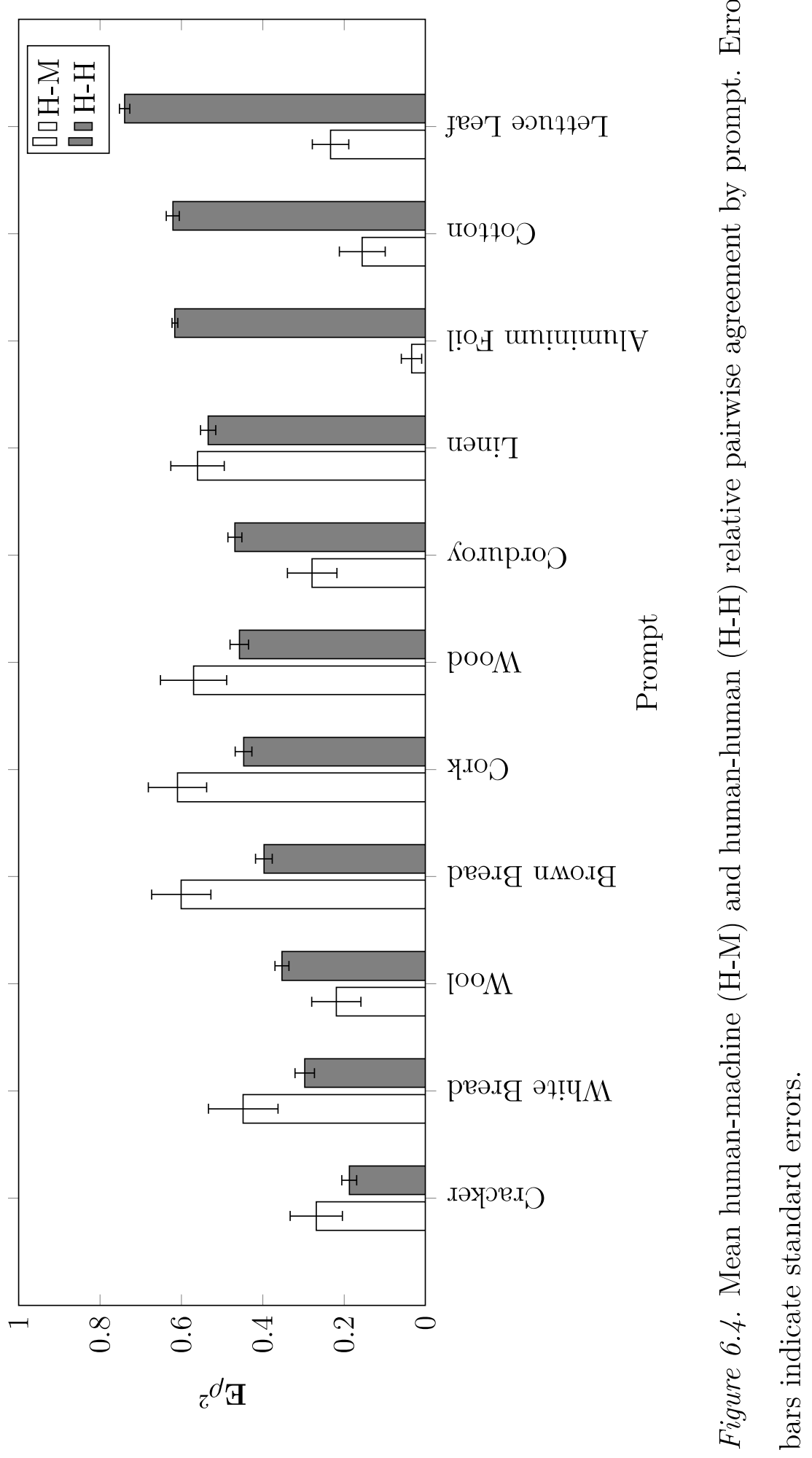




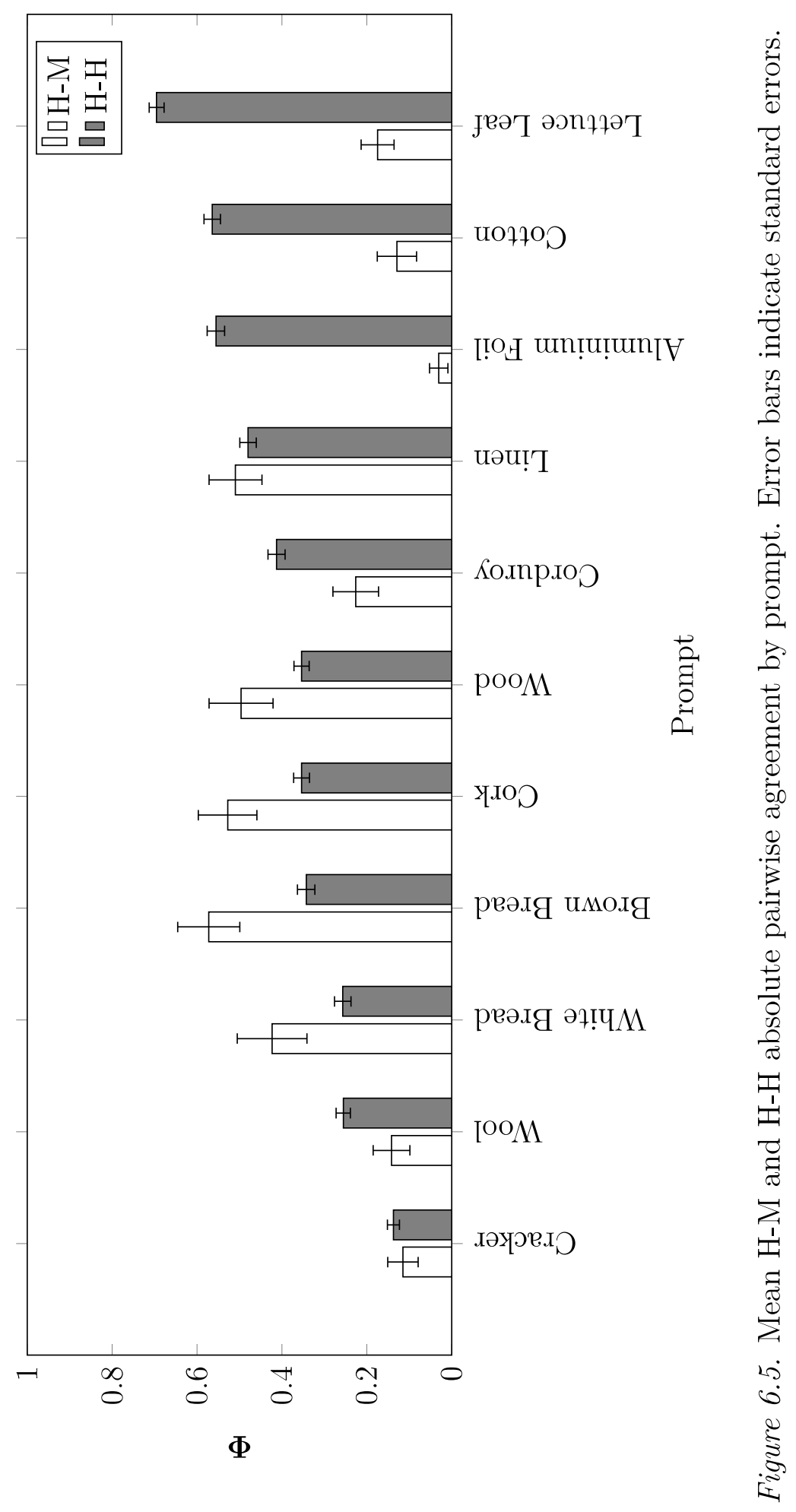


prompt by prompt basis relative to the difficulty of the prompt for $\mathrm{H}-\mathrm{H}$ pairs. We find that, in both figures, $\mathrm{H}-\mathrm{H}$ agreement scores tend to be low for prompts that show low acceptance rates in Figure 6.2 and that $\mathrm{H}-\mathrm{H}$ agreement scores tend to be high for prompts that show high acceptance rates in Figure 6.2. A striking observation is that for prompts where $\mathrm{H}-\mathrm{H}$ agreement is highest, $\mathrm{H}-\mathrm{M}$ agreement tends to be lowest. This suggests that images corresponding to these prompts are processed differently by the artificial assessor as compared to the human assessors. An equally striking observation is that $\mathrm{H}-\mathrm{M}$ pairs tend to perform above $\mathrm{H}-\mathrm{H}$ levels for most prompts excepting those for which $\mathrm{H}-\mathrm{H}$ agreement is highest.

\subsection{Discussion}

The present experiment compared human-machine and human-human agreement on a mirroring compliance assessment task for synthetic textures. The results obtained from this experiment are very preliminary, but promising, results for the approach to mirroring compliance assessment described in Chapter 4.

\subsubsection{Human Judgment Data}

First let us discuss the validity of human assessor judgments. Balas (2006) investigated the effects of various parameter settings for the Portilla-Simoncelli texture model (Portilla \& Simoncelli, 2000) on the discriminability of synthesized textures. His results reveal that coefficient correlations significantly contribute to texture discriminability at the preattentive stage. Omitting coefficient correlations during synthesis causes texture discriminability to increase. Balas found no similar effect for cross-scale phase statistics.

Recall that imaginer $\mathfrak{I}_{1}$ made use of the full Portilla-Simoncelli texture model 
parameter set, whereas imaginer $\mathfrak{I}_{2}$ omitted cross-scale phase statistics in texture image generation and imaginer $\mathfrak{I}_{3}$ omitted both coefficient correlations as well as cross-scale phase statistics. We observed that images sourced from $\mathfrak{I}_{1}$ and $\mathfrak{I}_{2}$ were rejected at nearly identical rates whereas images from $\mathfrak{I}_{3}$ were rejected at significantly higher rate. These observations agree with the findings in (Balas, 2006) and suggest that participants were able to perform the mirroring compliance assessment task as required.

However, an inspection of ground truth image acceptance rates for individual prompts revealed that images associated with some prompts (e.g., 'cracker') may not have been familiar or recognizable to human participants, whereas images associated with other prompts (e.g., 'cotton') appear to be easily identifiable to human participants. Prompts with low ground truth image acceptance rates are troubling. If human participants are unfamiliar with the application domain they cease to be qualified assessors. Nevertheless, human participants were able to discriminate images sourced from imaginers from those sourced from the ground truth dataset and they were also able to discriminate between imaginers, suggesting that test images were familiar enough that human assessors were able to carry out the mirroring compliance assessment task.

\subsubsection{Pairwise Comparisons}

The primary goal of the present experiment was to compare the representativeness of the artificial assessor to that of its human peers. It was found that human-machine agreement was near or above human-human agreement for both relative and absolute decisions. Notably, an inspection of the data revealed that human-machine pairs tended to outperform human-human pairs for absolute judgments. On the other hand, 
human-human pairs outperform human-machine pairs for relative judgments. These findings suggest that the artificial mirroring compliance assessor's judgments are reasonably representative of human judgment on a mirroring compliance assessment task for natural textures. That is to say, not only is the assessor able to discriminate imaginings from ground truth data, but that it does so in somewhat similar fashion to humans.

Looking at human-machine and human-human pairs on a prompt by prompt basis, we can see where the artificial assessor diverges from human assessors. First, on prompts where human-human pairwise agreement is highest, artificial assessor agreement is also lowest. This suggests that for these prompts, human assessors informed their judgments in way that differs from artificial assessors. On other prompts, human-machine pairs tended to have higher agreement scores than human-human pairs, though differences in scores were relatively small compared to prompts where human-human pairs outperformed human-machine pairs.

Since human-human agreement scores for prompts with low ground truth acceptance rates tended to be low whereas human-human agreement scores for prompts with high ground truth acceptance rates tended to be high, human-machine pair performance may partially be attributed to humans assessors' being presented with relatively unfamiliar or unrecognizable natural texture images. At the same time, human-machine pairs achieved high or comparable performance to human-human pairs on some prompts with relatively high ground truth image acceptance rates such as 'linen' suggesting that the assessor can compete with humans on images that are familiar or recognizable to humans. 


\subsubsection{Conclusions}

The artificial assessor developed for the present experiment performs in a manner that is comparable to human assessors, and there is some evidence that the assessor can compete with humans assessors on a mirroring compliance assessment task where humans are qualified assessors. These results constitute progress on the road to implementing functional automated evaluation procedures for artificial imagination systems.

The present experiment reveals challenges for artificial assessor development. The texture images that were used to construct the stimulus set for the experiment were drawn from a dataset designed to facilitate research in material categorization. Evidence was found that, though the stimulus images were images of natural textures, they were not the kind of natural texture images with which ordinary humans are well acquainted. This presents a problem for a strong interpretation of the results reported in this chapter, as the performance of the artificial assessor may partially be due poor performance on the part of human assessors owing to the fact that test images were unfamiliar or unrecognizable to human assessors.

The observation above is suggestive of a potentially deeper problem. The artificial assessor was trained on the same data from which the test images are sourced. In other words, the assessors' ground truth knowledge of natural textures differs from that of humans to the extent that the ground truth dataset is unrepresentative of human knowledge of natural visual textures. Perhaps this is why we observe that the artificial assessor is least representative of human assessors on prompts where human assessors agree the most. The lesson here is that if one is to develop artificial assessors similar in design to the artificial assessor discussed in this chapter, one needs to ensure 
that the assessor's ground truth knowledge matches its intended application.

To conclude, the results of the present experiment suggest that mirroring compliance assessment is a feasible assessment methodology for artificial imagination sys-

tems. As, with a simple assessor design, we were able to obtain mirroring compliance assessment results that were comparable to those of human assessors.

\subsection{Closing Remarks}

I presented and discussed results from the experiment decribed in the previous chapter. I found that an artificial mirroring compliance assessor was able to perform in a comparable manner to human assessors on a mirroring compliance assessment task for natural textures. The findings discussed in the present chapter are promising for the general imagination system evaluation framework proposed in this thesis. 


\section{Chapter 7 Conclusion}

The goal of the present work was to demonstrate that well-motivated artificial assessors for imagination systems can be developed, and to make a case that an evaluation methodology incorporating such artificial assessors will be an improvement on current practices in the field. To my knowledge, there has been no prior attempt to develop such an approach to imagination system evaluation.

Over the course of the project, a significant amount of theory was developed concerning imagination tasks and imagnation task demands. I defined imagination tasks as tasks in which imaginers must generate imaginings by elaborating on given imaginative prompts. I suggested that we analyze the process of elaboration as one of making elaborative choices, and I argued that it is these choices imagination systems researchers should evaluate. Then, following (Walton, 1990), I observed that elaborative choices seem to be governed by principles of generation. These considerations culminated in the following proposal: evaluation criteria for imagination systems can be developed through the identification and operationalization of applicable principles of generation.

Upon completing the theoretical discussion, I identified the mirroring principle (Gendler, 2003) as a salient principle of generation, and I proposed that compliance with the principle of mirroring could be measured in terms of classifier performance 
in a one-class classification setting. I piloted a mirroring compliance assessment procedure to evaluate some texture synthesis algorithms and I compared the automatic assessors to human assessors.

\subsection{General Discussion}

The implementation of a functioning mirroring compliance assessor is one of the major contributions of this work. It serves as a proof-of-concept for the proposed imagination system evaluation methodology and demonstrates the feasibility of the approach. It is notable that the artificial assessor was found to be reasonably representative of human assessor mirroring compliance judgments, despite the generality of the motivations behind its design.

The artificial assessor's design only took into account common common knowledge that a linear-non-linear-linear filter architecture is well suited for texture discrimination. All other design choices follow from theoretical and methodological considerations about imagination system evaluation. Notably, no effort was made to ensure that the assessor made use of biologically or psychologically plausible texture representations. There is potential for significant improvements to machine assessor performance through the incorporation of some design decisions that better model human perceptual processes.

However, it was also found that the artificial assessors' performance may be due, in part, to human assessors' being unfamiliar with experimental stimuli. It is difficult to conclude from the present study to what extent artificial assessor judgments are representative of human assessor judgments. However the present study constitutes significant progress toward the implementation of artificial assessors that are adequately representative of their human counterparts. 
One of the motivations behind the proposed approach to imagination system evaluation is that the use of human assessors in imagination system evaluation is resource intensive. It was suggested that in implementing artificial assessors, researchers could forgo the costs associated with recruiting and running human participants.

Ideally, an artificial mirroring compliance assessor would be representative enough to stand in for human assessors. However, it cannot be said that the assessor developed here is at such a level of performance. A question then arises as to the purported benefit of implementing artificial assessors for reducing the cost of running human participants - if assessors are not adequately representative, they cannot replace human assessors. However, it is worth noting that the present assessor is representative of human assessors to some extent. Though this means that we should not hope to replace human assessors with the present artificial assessor, the artificial assessor can nevertheless be used to prototype imagination systems. In other words, a useful application for an assessor with less-than-ideal representativeness may be to put it to use in rapidly evaluating imagination system prototypes. Such assessors could be used to facilitate the imagination system development process, without necessarily replacing other more conventional means of evaluation.

I have discussed the successes and limitations of the artificial assessor in connection with its performance on the mirroring compliance assessment task, but there is another dimension to the assessor that is equally important. Since the assessor is based on an explicit theory of imagination system correctness, the scores it assigns have a clear interpretation. If an imaginer is assessed as being poor by the artificial assessor, that imaginer has poor mirroring compliance. That is to say, the performance of an imagination system is interpretable in terms of a theory of imagination thanks 
to the assessor. This is a significant improvement over existing approaches to imagination system evaluation as existing approaches do not support similar generalization supporting interpretations.

In as much as demonstrating the feasibility of automatic imagination system evaluation procedures and making a case that such evaluation procedures can be considered improvements over existing approaches, the results of the present work are very promising. I would like to add a few comments concerning the methodological framework developed in earlier chapters. It is worth keeping in mind that the artificial assessor's score assignments support clear interpretations thanks to the fact that the proposed methodological framework facilitated the operationalization of an important constraint on imagination system behavior. This speaks to the promise of the proposed framework.

It is also worth noting that the proposed framework actually suggests a useful division of labor for the imagination systems research community. If one works within the proposed framework, one is faced with two notable tasks. The first of these tasks is to develop high-performing imagination systems, whereas the second task is to develop assessors for these imagination systems. Assessor development can be carried out independently from imagination system development. Furthermore, assessors can be standardized, facilitating their development and giving the community an opportunity to compete and to collaborate. The development of standardized assessors and the task of developing high-performance imagination systems are mutually beneficial lines of research. As high-performing imagination systems are developed, there will be incentive to develop more powerful imagination system assessors which, in turn will provide incentive for the development of still more powerful imagination systems. 


\subsection{Future Work}

Before closing, let me discuss some directions for future work. The present study implemented a mirroring compliance assessor for the domain of texture synthesis. It remains an open question whether the successes of the present artificial assessor will generalize to other domains. Texture images are relatively unstructured when compared to other data such as natural photographs or text. Future work should can focus on generalizing the present results to these domains.

One promising direction would be to replicate work on validating Coherencer (Vertolli \& Davies, 2013, 2014a, 2014b) within the mirroring compliance assessment framework. Coherencer models how humans select scene elements when given a mental imagery prompt denoting a single object such as 'cat'. As such, Coherencer uses simple symbolic representations. In generalizing results to domains where information is highly structured or symbolic, different artificial assessor designs might be required. For instance, a symbolic pattern-matching may be more suitable in some contexts making use of language-like representations.

Let me return to some remarks made in Chapter 4 . Recall that mirroring is not a strict principle of generation. Its constraints can be more or less relaxed depending on the specifics of an episode of imagination. Gendler (2003) calls this phenomenon disparity. The design of the mirroring compliance assessor relies on an operationalization of mirroring compliance that does not take disparity into account. Furthermore, current assessor design does not support mirroring assessments for unusual prompts such as requests to imagine blue apples. If prompts require imaginers to produce unusual imaginings, imaginers should not be punished for doing so. Practically, this would mean that an assessor would have to discount unusual features present in imag- 
inative prompts. The current mirroring compliance assessor design does not support such fine judgments. Thus, two other directions for future work is to make mirroring compliance assessors compatible with phenomena such as disparity and unusual prompts.

Finally, future research should aim to identify more principles of generation for operationalization. The present project was made possible thanks to the fact that an important principle of generation had already been identified in the literature. However, if the methodology proposed here is adopted, we will eventually have to refine our understanding of principles of generation. It should be noted that principles of generation can be established by convention. Walton (1990) gives the example of two friends who find themselves in a forest and decide to play a game where they consider tree stumps to be bears. This kind of principle of generation is remarkably different from the mirroring principle in that it strongly depends on contextual factors. I suspect implementing assessors for such principles will prove to be challenging. For now, we can focus on principles of generation that, like the principle of mirroring, have more general applicability. As our understanding of the mechanics of principles of generation improves, we can attempt to tackle more contextually sensitive principles. These suggestions are interesting as, in documenting and, effectively, modeling principles of generation, we stand to learn much about the functioning of human imagination. 


\section{Bibliography}

Aleksander, I., Dunmall, B., \& Del Frate, V. (1999). Neurocomputational Models of Visualisation: A Preliminary Report. In J. Mira \& J. V. Sánchez-Andrés (Eds.), Foundations and Tools for Neural Modeling: International Work-Conference on Artificial and Natural Neural Networks, IWANN'99 Alicante, Spain, June 2-4, 1999 Proceedings, Volume I (pp. 798-805). Berlin, Heidelberg: Springer Berlin Heidelberg. doi: 10.1007/BFb0098238

Balas, B. J. (2006). Texture synthesis and perception: Using computational models to study texture representations in the human visual system. Vision Research, 46, 299-309.

Breault, V., Ouellet, S., Somers, S., \& Davies, J. (2013). SOILIE: A computational model of 2D imagination. In R. West \& T. Stewart (Eds.), Proceedings of the 12th International Conference on Cognitive Modeling (ICCM2013) (pp. 95100). Ottawa, Carleton University.

Brennan, R. L. (2001). Generalizability Theory. New York: Springer-Verlag.

Brennan, R. L. (2003). Coefficients and Indices in Generalizability Theory (Tech. Rep.). Center for Advanced Studies in Measurement and Assessment.

Byrne, R. M. J. (2007). The Rational Imagination: How People Create Alternatives to Reality. Cambridge, MA: MIT Press.

Chella, A., Frixione, M., \& Gaglio, S. (2005). Planning by imagination in Cicerobot, 
a robot for museum tours. In AISB'05: Proceedings of the Symposium on Next Generation Approaches to Machine Consciousness Imagination, Development, Intersubjectivity and Embodiment (pp. 40-49).

Currie, G., \& Ravenscroft, I. (2002). Recreative Minds: Imagination in Philosophy and Psychology. OUP.

Dau, H. A., Ciesielski, V., \& Song, A. (2014). Anomaly Detection Using Replicator Neural Networks Trained on Examples of One Class. Simulated Evolution and Learning, 311-322.

Davies, J., Atance, C., \& Martin-Ordas, G. (2011). A framework and open questions on imagination in adults and children. Imagination, Cognition, and Personality, 31(1-2), 143-157.

Davis, C. E., \& Steinberg, S. M. (2006). Quantile estimation. In Encyclopedia of Statistical Sciences (Vol. 10). John Wiley \& Sons.

Fodor, J. (1994). Fodor's Guide to Mental Representation: The Intelligent Auntie's Vade-Mecum. In Stephen P. Stich and Ted A. Warfield (Ed.), Mental Representation: A Reader (chap. 1). Wiley-Blackwell. (Reprinted from Mind, 1985, $94[373], 76-100)$

Gagne, J., \& Davies, J. (2013). Visuo: A model of visuospatial instantiation of quantitative magnitudes. Knowledge Engineering Review, 1-20.

Galanter, P. (2010). The problem with evolutionary art is ... In European Conference on the Applications of Evolutionary Computation (Vol. 6025 LNCS, pp. 321$330)$.

Gaut, B. (2003). Creativity and imagination. The Creation of Art, 148-173.

Gendler, T. S. (2003). On the Relation Between Pretense and Belief. In M. Kieran 
\& D. M. Lopes (Eds.), Imagination Philosophy and the Arts (pp. 125-141). Routledge.

Gendler, T. S. (2013). Imagination. In E. N. Zalta (Ed.), The Stanford Encyclopedia of Philosophy (Fall 2013 ed.). The Metaphysics Research Lab, Center for the Study of Language and Information. Retrieved from http://plato.stanford.edu/ archives/fall2013/entries/imagination/

Hallin, N. J., Egbo, H., Ray, P., Soule, T., O’Rourke, M., \& Edwards, D. (2009). Enabling Autonomous Underwater Vehicles to Reason Hypothetically. In Mts/ieee biloxi - marine technology for our future: Global and local challenges, oceans 2009

Hawkins, S., He, H., Williams, G., \& Baxter, R. (2002). Outlier detection using replicator neural networks. Proceedings of the 4 th International Conference on Data Warehousing and Knowledge Discovery, 170-180.

Heath, D., Dennis, A., \& Ventura, D. (2015). Imagining Imagination: A Computational Framework Using Associative Memory Models and Vector Space Models. In Proceedings of the Sixth International Conference on Computational Creativity.

Hornby, G. S. (2003). Generative Representations for Computer-Automated Evolutionary Design. Intelligent Systems Division NASA(October 2003), 10.

Humberstone, I. L. (1992). Direction of fit. Mind, 101(401), 59-83.

Japkowicz, N., Jos, \& Gluck, M. a. (2000). Nonlinear Autoassociation is not Equivalent to PCA. Neural computation, 12(3), 531-545.

Johnson-Laird, P. N. (1987). Reasoning, imagining and creating. Bulletin of The British Psychological Society, 40, 121-129. 
Kosslyn, S. M. (1995). Mental Imagery. In S. M. Kosslyn \& D. N. Osherson (Eds.), An Invitation to Cognitive Science Volume 2: Visual Cognition. Cambridge, MA: MIT Press.

Kosslyn, S. M., \& Schwartz, S. P. (1977). A simulation of visual imagery. Cognitive Science, 1, 265-295.

Kosslyn, S. M., Thompson, W. L., Sukel, K. E., \& Alpert, N. M. (2005). Two types of image generation: Evidence from PET. Cognitive, Affective, and Behavioral Neuroscience, 5(1), 41-53.

Lewis, M. (2008). The art of artificial evolution: A handbook on evolutionary art and music. In J. J. Romero \& P. Machado (Eds.), (pp. 3-37). Springer.

Liu, J., Dong, J., Cai, X., Qi, L., \& Chantler, M. (2015). Visual perception of procedural textures: Identifying perceptual dimensions and predicting generation models. PLoS One, $10(6)$.

Lynch, C., Ashley, K., Pinkwart, N., \& Aleven, V. (2009). Concepts, Structures, and Goals: Redefining Ill-Definedness. International Journal of Artificial Intelligence in Education, 19(3), 253-266.

Mallikarjuna, P., Targhi, A. T., Fritz, M., Hayman, E., Caputo, B., \& Eklundh, J.-O. (2006). The KTH-TIPS2 database. Retrieved from http://www.nada.kth.se/ cvap/databases/kth-tips/kth-tips2.pdf

Morton, G. A. (2000). Machines with Imagination. Proceedings of the IEEE, 88(2), 283. (Reprinted from Proceedings of the IRE, 1962, 50[5], 661)

Moulton, S. T., \& Kosslyn, S. M. (2009). Imagining predictions: Mental imagery as mental emulation. Philosophical Transactions of the Royal Society of London B: Biological Sciences, 364(1521), 1273-1280. 
Murray, J. (2007). Visual recognition and inference using dynamic overcomplete sparse learning. Neural Computation, 19(9), 2301-2352.

Nichols, S., \& Stich, S. (2000). A cognitive theory of pretense. Cognition, 74, $115-147$.

Norton, D., Heath, D., \& Ventura, D. (2013). Finding creativity in an artificial artist. Journal of Creative Behavior, 47(2), 106-124.

Page, E. B. (1994). Computer Grading of Student Prose, Using Modern Concepts and Software. Journal of Experimental Education, 62(2), 127-142.

Pappas, T. N., Neuhoff, D. L., De Ridder, H., \& Zujovic, J. (2013). Image Analysis: Focus on Texture Similarity. Proceedings of the IEEE, 101(9), 2044-2057.

Portilla, J., \& Simoncelli, E. P. (2000). A Parametric Texture Model Based on Joint Statistics of Complex Wavelet Coefficients. International Journal of Computer Vision, 40(1), 49-71.

Rosenholtz, R. (2014). Texture Perception. In Oxford handbook of perceptual organization.

Roy, D., Hsiao, K.-Y., \& Mavridis, N. (2004). Mental imagery for a conversational robot. IEEE Transactions on Systems, Man and Cybernetics, Part B (Cybernetics), $34(3), 1374-1383$.

Setchi, R., Lagos, N., \& Froud, D. (2007). Computational Imagination: Research Agenda. In M. A. Orgun \& J. Thornton (Eds.), AI 200\%: Advances in Artificial Intelligence (pp. 387-393). Springer-Verlag Berlin Heidelberg.

Shavelson, R. J., \& Webb, N. M. (1991). Generalizability Theory: A Primer. Newbury Park, CA: Sage Publications.

Somers, S., Gagné, J., Astudillo, C., \& Davies, J. (2011). Using Semantic Similarity 
to Predict Angle and Distance of Objects in Images. In Proceedings of the 8th ACM Conference on Creativity \& Cognition (p. 217-222). Atlanta, Georgia.

Stening, J., Jacobsson, H., \& Ziemke, T. (2005). Abstraction of Sensorimotor Flow: Towards a Robot Model. In AISB'05: Proceedings of the Symposium on Next Generation Approaches to Machine Consciousness Imagination, Development, Intersubjectivity and Embodiment (pp. 50-58).

Stevenson, L. (2003). Twelve conceptions of imagination. British Journal of Aesthetics, 43(3), 238-259.

Tabachneck-Schijf, H. J. M., Leonardo, A. M., \& Simon, H. A. (1997). CaMeRa: A Computational Model of Multiple Representations. Cognitive Science, 21(3), 305-350.

Tax, D. M. J. (2001). One-class classification: Concept-learning in the absence of counter-examples (Unpublished doctoral dissertation). Delft University of Technology.

Thomee, B., Huiskes, M. J., Bakker, E., \& Lew, M. S. (2007). An Artificial Imagination for Interactive Search. In M. Lew, N. Sebe, T. S. Huang, \& E. M. Bakker (Eds.), Human-Computer Interaction: IEEE International Workshop, HCI 2007, Rio de Janeiro, Brazil, October 20, 200\%: Proceedings. Springer-Verlag Berlin Heidelberg.

Thomee, B., Huiskes, M. J., Bakker, E., \& Lew, M. S. (2008). Using an Artificial Imagination for Texture Retrieval. In Proceedings of the 19th International Conference on Pattern Recognition (ICPR 2008).

Togelius, J., Yannakakis, K., Stanley, K. O., \& Browne, C. (2011). Search-based procedural content generation: A taxonomy and survey. IEEE Transactions on 
Computational Intelligence and AI in Games, 3(3), 172-186.

Urmson, J. O. (1967). Memory and imagination. Mind, 76(301), 83-91.

Vertolli, M. O., Breault, V., Ouellet, S., Somers, S., Gagné, J., \& Davies, J. (2014).

Theoretical Assessment of the SOILIE Model of Human Imagination. In Proceedings of the 36th Annual Meeting of the Cognitive Science Society.

Vertolli, M. O., \& Davies, J. (2013). Visual imagination in context: Retrieving a coherent set of labels with Coherencer. In R. West \& T. Stewart (Eds.), Proceedings of the 12th International Conference on Cognitive Modeling (ICCM2013) (p. 263-268). Ottawa, Carleton University.

Vertolli, M. O., \& Davies, J. (2014a). Coherence in the Visual Imagination: Local Hill Search Outperforms Thagard's Connectionist Model. In Proceedings of the 36th Annual Meeting of the Cognitive Science Society.

Vertolli, M. O., \& Davies, J. (2014b). Coherence Net: A New Model of Generative Cognition. In Proceedings of the International Conference on Evolutionary Computation Theory and Applications (p. 308-313). Rome, Italy.

Vico, F. J., Veredas, F. J., Bravo, J. M., \& Almaraz, J. (1999). Automatic design synthesis with artificial intelligence techniques. Artificial Intelligence in Engineering, 13(3), 251-256.

Walton, K. L. (1990). Mimesis as Make-Believe: On the Foundations of the Representational Arts. Cambridge, MA: Harvard University Press.

Ward, T. B. (1994). Structured imagination: The role of category structure in exemplar generation. Cognitive Psychology, 27, 1-40.

Weisberg, D. S., \& Goodstein, J. (2009). What belongs in a fictional world? Journal of Cognition and Culture, 9, 69-78. 


\section{Appendix A Briefing Text and Comprehension Questions}

Briefing text and comprehension questions used during the collection of human assessor judgments are reproduced below. See Chapter 5.3 for procedural details.

The briefing text read as follows.

Imagine some kind of fabric. What color is it? How thick is it? How is it woven? If you have answers to these questions, you have created an imagining! That is to say, you have elaborated on the prompt above using your own knowledge.

Some computer programs can do something very similar to what you just did. We call computer programs that mimic imagination in this way artificial imagination systems.

These programs generate content (images, text etc.) from scratch based on some initial instructions (e.g. 'generate a cotton texture'). We call content created by such systems artificial imaginings.

In this study we ask you to help us validate an evaluation procedure for artificial imagination systems. Your task is to observe a number of images and to indicate, for each image, whether you think it is a photograph or an artificial imagining. 
The images that you will be observing are natural texture images. Natural textures are the patterns of colors and shapes that are characteristic of the surfaces of natural materials. The images will be black and white and they will be accompanied by the name of the material whose texture is depicted.

After participants read the briefing text, they had to answer the following questions correctly in order to proceed to experimental trials.

1. Which of the following counts as an artificial imagination system?

(a) A computer program that lets users write, edit and save text.

(b) A computer program that takes a scene description and generates an image of that scene.

(c) A computer program that takes some search terms and returns a list of websites that match those terms.

Answer: (b).

2. An image can be a photograph or an imagining. What distinguishes a photograph from an imagining?

(a) A photograph is an image created by the use of a camera whereas an imagining is created by an artificial imagination system.

(b) An imagining is an image created by the use of a camera whereas a photograph is created by an artificial imagination system.

(c) The two are impossible to tell apart. 
Answer: (a).

3. Which of the following is a natural texture image?

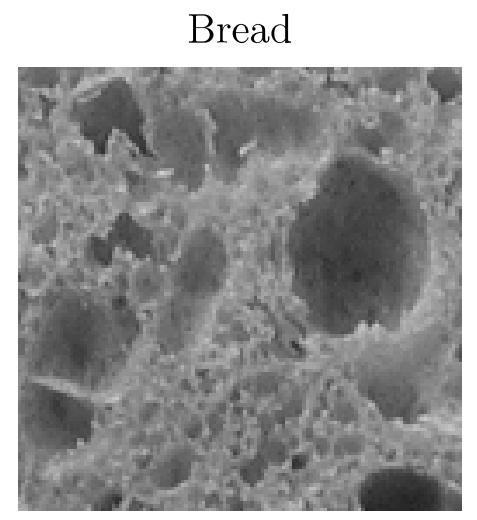

A
Bread

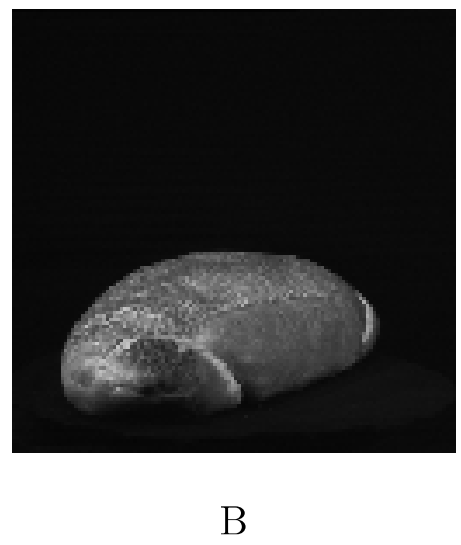

Answer: A. 